\title{
烷烃与氢过氧自由基氢提取反应类反应能垒与速率常数的精确计算
}

\author{
姚 倩 ${ }^{1} \quad$ 彭莉娟 $^{2}$ 李泽荣 ${ }^{1, *} \quad$ 李象远 $^{3}$ \\ ('四川大学化学学院, 成都 610064; ${ }^{2}$ 四川大学空天科学与工程学院, 成都 $6100655^{3}$ 四川大学化学工程学院, 成都 610065)
}

\begin{abstract}
摘要: 氢过氧自由基从烷烃分子中提取氢的反应是碳氢燃料中低温燃烧化学中非常重要的一类反应。本文用 等键反应方法计算了这一类反应的动力学参数。所有反应物、过渡态、产物的几何结构均在 $H F / 6-31+G(d)$ 水平下优化得到。以反应中的过渡态反应中心的几何结构守恒为判据, 该反应类可用等键反应处理。本文选 取了乙烷和氢过氧自由基的氢提取反应为参考反应, 其它反应作为目标反应, 用等键反应方法对目标反应在 $H F / 6-31+G(d)$ 水平的近似能垒和反应速率常数进行了校正。为了验证方法的可靠性, 选取 $\mathrm{C}_{5}$ 以下的烷烃分 子体系, 对等键反应方法校正结果和高精度 $\operatorname{CCSD}(\mathrm{T}) / \mathrm{CBS}$ 直接计算结果进行了比较, 最大绝对误差为 5.58 $\mathrm{kJ} \cdot \mathrm{mol}^{-1}$, 因此, 采用等键反应方法只需用低水平 $\mathrm{HF}$ 从头算方法就可以再现高精度 $\operatorname{CCSD}(\mathrm{T}) / \mathrm{CBS}$ 计算结 果, 从而解决了该反应类中大分子体系的能垒的精确计算。本文的研究为碳氢化合物中低温燃烧模拟中重要 的烷烃与氢过氧自由基氢提取反应提供了准确的动力学参数。
\end{abstract}

关键词: 反应类等键反应方法; 反应能垒; 速率常数; 烷烃氢提取; 中低温燃烧 中图分类号: 0643

\section{Accurate Calculation of the Energy Barriers and Rate Constants of Hydrogen Abstraction from Alkanes by Hydroperoxyl Radical}

\author{
YAO Qian ${ }^{1} \quad$ PENG Li-Juan ${ }^{2} \quad$ LI Ze-Rong ${ }^{1, *} \quad$ LI Xiang-Yuan ${ }^{3}$ \\ ( ${ }^{1}$ College of Chemistry, Sichuan University, Chengdu 610064, P. R. China; ${ }^{2}$ School of Aeronautics \& Astronautics, Sichuan \\ University, Chengdu 610065, P. R. China; ${ }^{3}$ School of Chemical Engineering, Sichuan University, Chengdu 610065, P. R. China)
}

\begin{abstract}
Hydrogen abstraction from alkanes by hydroperoxyl radical is an important reaction class in the combustion of hydrocarbon fuel, particularly at low and intermediate temperature regimes. In this study, kinetic parameters for this reaction class are calculated using the isodesmic reaction method based on conservation of geometric structures for the reaction center of the transition states. The geometries for all the reactants, transition states, and products are optimized at the $\mathrm{HF} / 6-31+\mathrm{G}(d)$ level. Hydrogen abstraction from ethane by hydroperoxyl radical is chosen as the reference reaction; other reactions are target reactions. The isodesmic reaction method is used to correct the approximate energy barriers and rate constants of the target reactions at the $\mathrm{HF} / 6-31+\mathrm{G}(d)$ level. To validate the reliability of the isodesmic reaction method, the energy barriers calculated by the isodesmic reaction method and at a high level of CCSD(T)/CBS for alkanes containing less than five carbon atoms are compared. The maximum absolute difference of energy barriers between the isodesmic reaction method and $\operatorname{CCSD}(\mathrm{T}) /$ CBS method is $5.58 \mathrm{~kJ} \cdot \mathrm{mol}^{-1}$. Therefore, after correction, using the isodesmic reaction method, the low-level $\mathrm{HF}$ method can reproduce the high-level CCSD(T)/CBS calculated energy barriers. Thus, we have solved the problem of accurately calculating energy barriers for large molecular systems in this reaction class. The present work provides accurate kinetic parameters for hydrogen abstraction from alkanes by hydroperoxyl radical, which are important for combustion modeling at low and intermediate temperature regimes.
\end{abstract}

Received: November 3, 2016; Revised: January 9, 2017; Published online: January 9, 2017.

${ }^{*}$ Corresponding author. Email: lizerong@scu.edu.cn; Tel: +86-28-85406139.

The project was supported by the National Natural Science Foundation of China (91441114).

国家自然科学基金(91441114)资助项目

(C) Editorial office of Acta Physico-Chimica Sinica 
Key Words: Reaction class isodesmic reaction method; Energy barrier of reaction; Rate constant; Hydrogen abstraction of alkanes; Combustion at low and intermediate temperature regimes

\section{1 引言}

目前, 由于碳氢燃料的使用越来越广泛, 如 何提高燃烧效率和减少污染物排放等是发动机燃 烧室设计需要解决的问题。大量的实验技术应用 于碳氢燃料的燃烧过程研究, 与此同时, 随着量 子化学的发展, 碳氢燃料在高温条件下的燃烧模 拟研究已经取得了很大成就 ${ }^{1,2}$, 发展建立了大量的 单组分碳氢燃料和不同混合燃料的替代燃烧模 型, 可以较好地再现高温实验结果。但是在中低 温条件下, 由于包含的反应种类非常多, 产生的 物种数量也较为庞大, 许多反应类型缺乏可靠的 动力学参数, 中低温然烧化学有待进一步完善。 氢过氧自由基从烷烃提取氢原子反应是中低温燃 烧化学中重要的一个反应类型, 对点火起着至关 重要的作用。然而通过实验的手段来测量这一类 基元反应的反应速率常数是很困难的, 一方面这 类反应能垒较高因而速率常数较低, 另一方面通 过升高温度来增加速率常数会导致氢过氧自由基 的分解。目前仅有少数烷烃与氢过氧自由基氢提 取反应的动力学实验数据有文献报道, 这些数据 是通过间接测量技术得到, 且适用温度范围很 窄 ${ }^{3-7}$, 缺乏对该反应类的系统研究。

对这类反应中从 $\mathrm{C}_{1}$ 到 $\mathrm{C}_{4}$ 的烷烃与氢过氧自由 基氢提取反应的计算研究, 文献已有报道。 Carstensen 等 ${ }^{8}$ 在 CBS-QB3 水平下计算了从 $\mathrm{C}_{1}$ 到 $\mathrm{C}_{4}$ 的烷基与氢过氧自由基氢提取反应的反应能垒, 通过传统过渡态理论得到了这些反应的反应速率 常数。Aguilera 等 ${ }^{9}$ 采用 $\operatorname{CCSD}(\mathrm{T}) / \mathrm{cc}-\mathrm{pVTZ}$ 方法计 算了从 $\mathrm{C}_{1}$ 到 $\mathrm{C}_{4}$ 的烷基与氢过氧自由基氢提取反应 的反应能垒, 通过传统过渡态理论计算了这些反 应的反应速率常数。文献报道的体系均为 $\mathrm{C}_{4}$ 及以 下体系, 目前的量子化学高精度计算方法只能局 限于较少碳原子数的烷烃分子体系。

Truong 等 ${ }^{10-16}$ 将反应类的概念引入过渡态理论 中, 提出了反应类过渡态理论 (RC-TST)。该理论 认为同一类反应中所有反应具有相同的活性中 心。将同一类反应中最小反应体系定义为参考反 应, 其余反应皆为目标反应。Truong 等研究发 现, 参考反应与目标反应的能垒差随从头算理论 方法变化很小, 即通过低水平的从头算理论方法 可得到较高精度大分子体系的动力学参数。但是
他们并没有解释参考反应与目标反应间的能垒差 随从头算理论方法变化很小的原因。在我们的前 期研究中, 我们对反应类过渡态理论提供了解 释, 将等键反应的定义推广到了含非典型化学键 的过渡态, 用于精确计算反应类目标反应能垒及 速率常数 17,18 , 并用于对密度泛函理论(DFT)水平 计算的反应能垒和速率常数进行校正, 得到了较 好的结果 ${ }^{19}$ 。Hartree-Fock (HF) 方法 ${ }^{20}$ 是最低级别 的从头算方法, HF 方法的计算消耗比一般的 DFT 方法要小, 可用于更大分子体系的计算, 但由于 没有考虑电子相关能, 一般不能用于计算化学反 应的能量。本文的目的是通过将等键反应理论与 过渡态理论相结合, 以氢过氧自由基从烷烃分子 中提取氢反应为研究对象, 解决更大分子体系动 力学参数的精确计算问题。

\section{2 计算方法}

根据传统过渡态理论, 双分子反应如下:

$\mathrm{X}+\mathrm{Y} \rightarrow$ products

此反应的速率常数的表达式为:

$$
k=\kappa\left(k_{\mathrm{B}} \mathrm{T} / h\right)\left(Q^{\ddagger} / Q_{\mathrm{x}} Q_{\mathrm{Y}}\right) \exp \left(-\Delta V^{\ddagger} / R T\right)
$$

其中, $\kappa$ 为隧穿因子, $k_{\mathrm{B}}$ 是玻尔兹曼常数, $h$ 是普 朗克常数, $T$ 为温度, $R$ 是理想气体常数, $Q^{\neq}$是过 渡态的配分函数, $Q_{\mathrm{X}} 、 Q_{\mathrm{Y}}$ 分别是反应物 $\mathrm{X}$ 和反应 物 $\mathrm{Y}$ 的配分函数, $\Delta V^{*}$ 是反应的能垒。以上配分函 数只包含振动、转动和平动的贡献, 仅与优化得 到的振动频率和几何结构有关, 而与单点能无 关, 因此单点能的计算级别只影响反应的能垒。

设 $\mathrm{P}$ 为参考反应, $\mathrm{T}$ 为目标反应, 根据等键反 应的方法 ${ }^{18}$ 目标反应势能如下:

$$
\begin{aligned}
& \Delta V_{\mathrm{T}}^{\neq \prime}=\Delta V_{\mathrm{T}}^{\neq}+\Delta \Delta V_{\mathrm{P}}^{\neq} \\
& \Delta \Delta V_{\mathrm{P}}^{\neq}=\Delta V_{\mathrm{P}}^{\neq \prime}-\Delta V_{\mathrm{P}}^{\neq}
\end{aligned}
$$

其中, $\neq$ 为表示过渡态的符号, $\Delta V_{\mathrm{P}}^{ \pm}$和 $\Delta V_{\mathrm{P}}^{ \pm}$分别为 高级别从头算理论方法和低级别从头算理论方法 计算得到的参考反应的反应能垒， $\Delta \Delta V_{\mathrm{P}}^{\neq}$为参考反 应能垒校正值。 $\Delta V_{\mathrm{T}}^{\neq}$为低级别从头算理论方法计算 得到的目标反应的反应能垒, $\Delta V_{\mathrm{T}}^{\neq}$为通过反应能垒 校正得到的高精度目标反应的反应能垒。目标反 应的精确速率常数 $k^{\prime}$ 可通过目标反应的速率常数 $k$ 与参考反应的能垒校正项得到:

$$
k^{\prime}=k \exp \left[\left(-\Delta \Delta V_{\mathrm{P}}^{\ddagger}\right) / R T\right]
$$


本文选取了 25 个烷烃分子与氢过氧自由基氢 提取反应作为研究对象, 反应列于表 1 中。

这些反应涉及到的所有物种的几何结构优 化、频率计算及低级别单点能计算都是在 HF/6$31+\mathrm{G}(d)$ 水平下进行, 采用内禀反应坐标(IRC)理论 对所有反应的过渡态进行了确认。频率校正因子为 0.924 , 零点能校正因子为 $0.911^{21}$ 。精确单点能采 用高精度 $\operatorname{CCSD}(\mathrm{T}) / \mathrm{cc}-\mathrm{pV} X \mathrm{Z}(\mathrm{X}=\mathrm{D}, \mathrm{T}, \mathrm{Q})$ 方法外推 到完全基组法(即 $\operatorname{CCSD}(\mathrm{T}) / \mathrm{CBS}$ 方法) ${ }^{22-26}$ 计算得到:

$$
\begin{aligned}
& E_{\mathrm{tot}}=E_{\infty}^{\mathrm{HF}}+E_{\infty}^{\mathrm{corr}} \\
& E_{X}^{\mathrm{HF}}=E_{\infty}^{\mathrm{HF}}+B \mathrm{e}^{-\alpha X} \\
& E_{\infty}^{\mathrm{corr}}=E_{X}^{\mathrm{corr}}+A /(X+1 / 2)^{3}
\end{aligned}
$$

其中, cc-pVDZ、cc-pVTZ 和 cc-pVQZ 基组中 $X$ 分 别等于 $2 、 3$ 和 $4, A 、 B 、 \alpha$ 都是常数, $E_{\infty}^{\mathrm{HF}}$ 通过 $\mathrm{HF} /$ cc-pVXZ $(X=\mathrm{D}, \mathrm{T}, \mathrm{Q})$ 外推得到, $E_{\infty}^{\mathrm{corr}}$ 通过 CCSD $(\mathrm{T}) / \mathrm{cc}-\mathrm{pV} X \mathrm{Z}(X=\mathrm{D}, \mathrm{T})$ 外推得到。所有的从头算计 算都在 Gaussian 09 程序 ${ }^{27}$ 上完成。

\section{3 结果与讨论}

\section{1 过渡态几何中心结构}

表 1 烷烃分子与氢过氧自由基氢提取反应方程式

\begin{tabular}{|c|c|}
\hline No. & Reaction \\
\hline $\mathrm{R}_{1}$ & $\mathrm{CH}_{4}+\mathrm{HO}_{2} \cdot \rightarrow \mathrm{CH}_{3}+\mathrm{H}_{2} \mathrm{O}_{2}$ \\
\hline $\mathrm{R}_{2}$ & $\mathrm{C}_{2} \mathrm{H}_{6}+\mathrm{HO}_{2} \cdot \rightarrow \mathrm{C}_{2} \mathrm{H}_{5}+\mathrm{H}_{2} \mathrm{O}_{2}$ \\
\hline $\mathrm{R}_{3}$ & $\mathrm{C}_{3} \mathrm{H}_{8}+\mathrm{HO}_{2} \cdot \rightarrow n-\mathrm{C}_{3} \mathrm{H}_{7} \cdot+\mathrm{H}_{2} \mathrm{O}_{2}$ \\
\hline $\mathrm{R}_{4}$ & $\mathrm{C}_{3} \mathrm{H}_{8}+\mathrm{HO}_{2} \cdot \rightarrow i-\mathrm{C}_{3} \mathrm{H}_{7} \cdot+\mathrm{H}_{2} \mathrm{O}_{2}$ \\
\hline $\mathrm{R}_{5}$ & $n-\mathrm{C}_{4} \mathrm{H}_{10}+\mathrm{HO}_{2} \cdot \rightarrow n-\mathrm{C}_{4} \mathrm{H}_{9} \cdot+\mathrm{H}_{2} \mathrm{O}_{2}$ \\
\hline $\mathrm{R}_{6}$ & $n-\mathrm{C}_{4} \mathrm{H}_{10}+\mathrm{HO}_{2} \cdot \rightarrow s-\mathrm{C}_{4} \mathrm{H}_{9} \cdot+\mathrm{H}_{2} \mathrm{O}_{2}$ \\
\hline $\mathrm{R}_{7}$ & $i-\mathrm{C}_{4} \mathrm{H}_{10}+\mathrm{HO}_{2} \cdot \rightarrow i-\mathrm{C}_{4} \mathrm{H}_{9} \cdot+\mathrm{H}_{2} \mathrm{O}_{2}$ \\
\hline $\mathrm{R}_{8}$ & $i-\mathrm{C}_{4} \mathrm{H}_{10}+\mathrm{HO}_{2} \cdot \rightarrow t-\mathrm{C}_{4} \mathrm{H}_{9} \cdot+\mathrm{H}_{2} \mathrm{O}_{2}$ \\
\hline $\mathrm{R}_{9}$ & $t-\mathrm{C}_{5} \mathrm{H}_{12}+\mathrm{HO}_{2} \cdot \rightarrow\left(\mathrm{CH}_{3}\right)_{3} \mathrm{CCH}_{2} \cdot+\mathrm{H}_{2} \mathrm{O}_{2}$ \\
\hline $\mathrm{R}_{10}$ & $n-\mathrm{C}_{5} \mathrm{H}_{12}+\mathrm{HO}_{2} \cdot \rightarrow \mathrm{CH}_{3}\left(\mathrm{CH}_{2}\right)_{3} \mathrm{CH}_{2} \cdot+\mathrm{H}_{2} \mathrm{O}_{2}$ \\
\hline $\mathrm{R}_{11}$ & $n-\mathrm{C}_{5} \mathrm{H}_{12}+\mathrm{HO}_{2} \cdot \rightarrow \mathrm{CH}_{3}\left(\mathrm{CH}_{2}\right)_{2} \mathrm{CH} \cdot \mathrm{CH}_{3}+\mathrm{H}_{2} \mathrm{O}_{2}$ \\
\hline $\mathrm{R}_{12}$ & $n-\mathrm{C}_{5} \mathrm{H}_{12}+\mathrm{HO}_{2} \cdot \rightarrow \mathrm{CH}_{3} \mathrm{CH}_{2} \mathrm{CH} \cdot \mathrm{CH}_{2} \mathrm{CH}_{3}+\mathrm{H}_{2} \mathrm{O}_{2}$ \\
\hline $\mathrm{R}_{13}$ & $i-\mathrm{C}_{5} \mathrm{H}_{12}+\mathrm{HO}_{2} \cdot \rightarrow \mathrm{CH}_{3} \mathrm{CH}_{2} \mathrm{CH}\left(\mathrm{CH}_{3}\right) \mathrm{CH}_{2} \cdot+\mathrm{H}_{2} \mathrm{O}_{2}$ \\
\hline $\mathrm{R}_{14}$ & $i-\mathrm{C}_{5} \mathrm{H}_{12}+\mathrm{HO}_{2} \cdot \rightarrow \mathrm{CH}_{3} \mathrm{CH}_{2} \mathrm{C} \cdot\left(\mathrm{CH}_{3}\right) \mathrm{CH}_{3}+\mathrm{H}_{2} \mathrm{O}_{2}$ \\
\hline $\mathrm{R}_{15}$ & $i-\mathrm{C}_{5} \mathrm{H}_{12}+\mathrm{HO}_{2} \cdot \rightarrow \mathrm{CH}_{3} \mathrm{CH} \cdot \mathrm{CH}\left(\mathrm{CH}_{3}\right) \mathrm{CH}_{3}+\mathrm{H}_{2} \mathrm{O}_{2}$ \\
\hline $\mathrm{R}_{16}$ & $i-\mathrm{C}_{5} \mathrm{H}_{12}+\mathrm{HO}_{2} \cdot \rightarrow \mathrm{CH}_{2} \cdot \mathrm{CH}_{2} \mathrm{CH}\left(\mathrm{CH}_{3}\right) \mathrm{CH}_{3}+\mathrm{H}_{2} \mathrm{O}_{2}$ \\
\hline $\mathrm{R}_{17}$ & $n-\mathrm{C}_{6} \mathrm{H}_{14}+\mathrm{HO}_{2} \cdot \rightarrow \mathrm{CH}_{3}\left(\mathrm{CH}_{2}\right)_{4} \mathrm{CH}_{2} \cdot+\mathrm{H}_{2} \mathrm{O}_{2}$ \\
\hline $\mathrm{R}_{18}$ & $n-\mathrm{C}_{6} \mathrm{H}_{14}+\mathrm{HO}_{2} \cdot \rightarrow \mathrm{CH}_{3}\left(\mathrm{CH}_{2}\right)_{3} \mathrm{CH}_{2} \cdot \mathrm{CH}_{3}+\mathrm{H}_{2} \mathrm{O}_{2}$ \\
\hline $\mathrm{R}_{19}$ & $i-\mathrm{C}_{6} \mathrm{H}_{14}+\mathrm{HO}_{2} \cdot \rightarrow \mathrm{CH}_{3}\left(\mathrm{CH}_{2}\right)_{2} \mathrm{C} \cdot\left(\mathrm{CH}_{3}\right)_{2}+\mathrm{H}_{2} \mathrm{O}_{2}$ \\
\hline $\mathrm{R}_{20}$ & $n-\mathrm{C}_{7} \mathrm{H}_{16}+\mathrm{HO}_{2} \cdot \rightarrow \mathrm{CH}_{3}\left(\mathrm{CH}_{2}\right)_{5} \mathrm{CH}_{2} \cdot+\mathrm{H}_{2} \mathrm{O}_{2}$ \\
\hline $\mathrm{R}_{21}$ & $n-\mathrm{C}_{7} \mathrm{H}_{16}+\mathrm{HO}_{2} \cdot \rightarrow \mathrm{CH}_{3}\left(\mathrm{CH}_{2}\right)_{4} \mathrm{CH}_{2} \cdot \mathrm{CH}_{3}+\mathrm{H}_{2} \mathrm{O}_{2}$ \\
\hline $\mathrm{R}_{22}$ & $i-\mathrm{C}_{7} \mathrm{H}_{16}+\mathrm{HO}_{2} \cdot \rightarrow \mathrm{CH}_{3}\left(\mathrm{CH}_{2}\right)_{3} \mathrm{C} \cdot\left(\mathrm{CH}_{3}\right)_{2}+\mathrm{H}_{2} \mathrm{O}_{2}$ \\
\hline $\mathrm{R}_{23}$ & $n-\mathrm{C}_{8} \mathrm{H}_{18}+\mathrm{HO}_{2} \cdot \rightarrow \mathrm{CH}_{3}\left(\mathrm{CH}_{2}\right)_{6} \mathrm{CH}_{2} \cdot+\mathrm{H}_{2} \mathrm{O}_{2}$ \\
\hline $\mathrm{R}_{24}$ & $n-\mathrm{C}_{8} \mathrm{H}_{18}+\mathrm{HO}_{2} \cdot \rightarrow \mathrm{CH}_{3}\left(\mathrm{CH}_{2}\right)_{5} \mathrm{CH}_{2} \cdot \mathrm{CH}_{3}+\mathrm{H}_{2} \mathrm{O}_{2}$ \\
\hline $\mathrm{R}_{25}$ & $i-\mathrm{C}_{8} \mathrm{H}_{18}+\mathrm{HO}_{2} \cdot \rightarrow \mathrm{CH}_{3}\left(\mathrm{CH}_{2}\right)_{4} \mathrm{C} \cdot\left(\mathrm{CH}_{3}\right)_{2}+\mathrm{H}_{2} \mathrm{O}_{2}$ \\
\hline
\end{tabular}

Table 1 Chemical reaction equations of hydrogen abstraction from alkanes by the hydroperoxyl radical
用 $\mathrm{HF} / 6-31+\mathrm{G}(d)$ 方法优化后的过渡态反应中 心所涉及到的原子、取代基、键长及键角编号如 图 1 所示。其中 $d_{1} 、 d_{2}$ 为键长, $A_{1} 、 A_{2}$ 为键角, $\mathrm{R}_{\mathrm{a}} 、 \mathrm{R}_{\mathrm{b}} 、 \mathrm{R}_{\mathrm{c}}$ 代表过渡态中的氢或烷基取代基。

$\mathrm{HF} / 6-31+\mathrm{G}(d)$ 方法优化得到的各反应的反应中 心过渡态几何结构参数列于表 2 。由表 2 可以看 出, 这一类反应的过渡态反应中心的几何结构相 差较小, 将断裂的单键 $d_{1}$ 和将形成的单键 $d_{2}$ 的最大 绝对误差分别为 0.0034 和 $0.0042 \mathrm{~nm}$, 键角 $A_{1}$ 和 $A_{2}$ 的最大绝对误差分别为 $3.9^{\circ}$ 和 $0.4^{\circ}$ 。其中最小反应

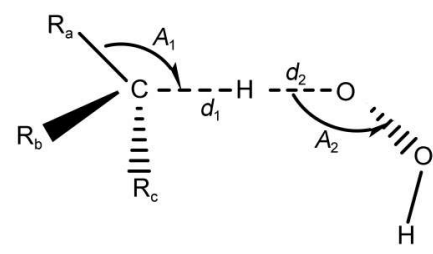

图 1 过渡态反应中心的几何结构

Fig.1 Geometry structures for the reaction center of the transition states

表 2 过渡态反应中心优化后的几何参数

Table 2 Optimized geometrical parameters of reaction center of transition states

\begin{tabular}{lcccc}
\hline $\mathrm{TS}$ & $d_{1} / \mathrm{nm}$ & $A_{1} /\left(^{\circ}\right)$ & $d_{2} / \mathrm{nm}$ & $A_{2} /\left(^{\circ}\right)$ \\
\hline $\mathrm{TS}_{1}$ & 0.1351 & 103.6 & 0.1173 & 104.1 \\
$\mathrm{TS}_{2}$ & 0.1337 & 102.0 & 0.1188 & 103.9 \\
$\mathrm{TS}_{3}$ & 0.1339 & 102.2 & 0.1187 & 104.0 \\
$\mathrm{TS}_{4}$ & 0.1327 & 104.8 & 0.1200 & 103.9 \\
$\mathrm{TS}_{5}$ & 0.1338 & 102.1 & 0.1188 & 104.0 \\
$\mathrm{TS}_{6}$ & 0.1326 & 104.2 & 0.1202 & 103.9 \\
$\mathrm{TS}_{7}$ & 0.1340 & 102.3 & 0.1189 & 103.9 \\
$\mathrm{TS}_{8}$ & 0.1319 & 103.3 & 0.1211 & 103.8 \\
$\mathrm{TS}_{9}$ & 0.1342 & 102.2 & 0.1189 & 104.2 \\
$\mathrm{TS}_{10}$ & 0.1338 & 102.1 & 0.1188 & 104.0 \\
$\mathrm{TS}_{11}$ & 0.1326 & 104.2 & 0.1202 & 103.9 \\
$\mathrm{TS}_{12}$ & 0.1329 & 104.7 & 0.1204 & 104.0 \\
$\mathrm{TS}_{13}$ & 0.1339 & 101.4 & 0.1190 & 104.2 \\
$\mathrm{TS}_{14}$ & 0.1317 & 102.7 & 0.1215 & 103.9 \\
$\mathrm{TS}_{15}$ & 0.1327 & 104.5 & 0.1204 & 103.8 \\
$\mathrm{TS}_{16}$ & 0.1337 & 101.7 & 0.1189 & 104.0 \\
$\mathrm{TS}_{17}$ & 0.1338 & 102.1 & 0.1188 & 104.0 \\
$\mathrm{TS}_{18}$ & 0.1329 & 105.3 & 0.1202 & 104.0 \\
$\mathrm{TS}_{19}$ & 0.1321 & 103.4 & 0.1213 & 103.8 \\
$\mathrm{TS}_{20}$ & 0.1338 & 101.8 & 0.1188 & 104.0 \\
$\mathrm{TS}_{21}$ & 0.1329 & 105.3 & 0.1202 & 104.0 \\
$\mathrm{TS}_{22}$ & 0.1321 & 103.4 & 0.1213 & 103.9 \\
$\mathrm{TS}_{23}$ & 0.1338 & 102.1 & 0.1189 & 104.0 \\
$\mathrm{TS}_{24}$ & 0.1329 & 105.3 & 0.1202 & 104.0 \\
\hline & 0.1321 & 103.9 & 0.1213 & 103.9 \\
\hline & 0.0034 & 3.9 & 0.0042 & 0.4 \\
\hline & & & & \\
\hline
\end{tabular}

${ }^{a}$ maximum absolute error 
$\mathrm{R}_{1}$ 与其他反应的键长相差相对较大, 因此我们选 取了反应 $\mathrm{R}_{2}$ 作为参考反应。任意目标反应与参考 反应 $\mathrm{R}_{2}$ 的代数差反应中的过渡态反应中心的几何 结构是守恒的, 由体系中任意目标反应与参考反 应 $\mathrm{R}_{2}$ 的差所构建的反应即为等键反应。

\section{2 反应能垒}

本文用等键反应方法对目标反应在 $\mathrm{HF} / 6-31+\mathrm{G}$ (d)水平的近似能垒进行了校正。为了验证方法的 可靠性, 选取 $\mathrm{C}_{5}$ 以下的分子体系, 用高精度 CCSD (T)/CBS 方法进行了精确计算, 外推数据 $E_{\infty}^{\mathrm{HF}}$ 和 $E_{\infty}^{\mathrm{corr}}$ 见表 S1 (见 Supporting Information), 其结果与直接 采用 HF 方法计算及用等键反应方法校正得到的反 应能垒比较列于表 3 。从表 3 可以看出, HF 方法和 $\mathrm{CCSD}(\mathrm{T}) / \mathrm{CBS}$ 方法计算反应能垒的平均绝对误差 高达 $90.19 \mathrm{~kJ} \cdot \mathrm{mol}^{-1}$, 远大于等键反应方法和 CCSD (T)/CBS 方法计算反应能垒的平均绝对误差 2.96 $\mathrm{kJ} \cdot \mathrm{mol}^{-1}$ 。 $\mathrm{HF}$ 方法和 $\mathrm{CCSD}(\mathrm{T}) / \mathrm{CBS}$ 方法计算反应 能垒的最大绝对误差为 $92.81 \mathrm{~kJ} \cdot \mathrm{mol}^{-1}$, 仍然远大 于等键反应方法和 $\mathrm{CCSD}(\mathrm{T}) / \mathrm{CBS}$ 方法计算反应能 垒的最大绝对误差 $5.58 \mathrm{~kJ} \cdot \mathrm{mol}^{-1}$ 。用等键反应方法 校正过后的结果已在化学反应精度内, 表明烷烃 与氢过氧自由基氢提取反应用 HF 方法计算经等键

\section{表3 HF 方法校正前后能垒与 $\mathrm{CCSD}(\mathrm{T}) / \mathrm{CBS}$ 结果比较 Table 3 Comparison of HF energy barriers before and after validation with the $\mathrm{CCSD}(\mathrm{T}) / \mathrm{CBS}$ results}

\begin{tabular}{|c|c|c|c|c|c|}
\hline \multirow{2}{*}{ Reaction- } & \multicolumn{5}{|c|}{$\Delta V /\left(\mathrm{kJ} \cdot \mathrm{mol}^{-1}\right)$} \\
\hline & $\operatorname{CCSD}(\mathrm{T}) / \mathrm{CBS}$ & $\mathrm{HF}$ & $\mathrm{IRM}^{\mathrm{a}}$ & $\mathrm{AD}_{\mathrm{HF}}{ }^{\mathrm{b}}$ & $\mathrm{AD}_{\mathrm{IRM}}{ }^{\mathrm{c}}$ \\
\hline $\mathrm{R}_{1}$ & 108.26 & 191.12 & - & - & - \\
\hline $\mathrm{R}_{2}$ & 91.74 & 178.97 & - & - & - \\
\hline $\mathrm{R}_{3}$ & 91.96 & 179.66 & 92.43 & 87.69 & 0.46 \\
\hline $\mathrm{R}_{4}$ & 80.34 & 169.73 & 82.50 & 89.39 & 2.16 \\
\hline $\mathrm{R}_{5}$ & 91.07 & 179.31 & 92.08 & 88.24 & 1.01 \\
\hline $\mathrm{R}_{6}$ & 80.91 & 172.64 & 85.42 & 91.73 & 4.50 \\
\hline $\mathrm{R}_{7}$ & 89.51 & 179.63 & 92.41 & 90.12 & 2.89 \\
\hline $\mathrm{R}_{8}$ & 69.21 & 160.93 & 73.70 & 91.72 & 4.49 \\
\hline $\mathrm{R}_{9}$ & 90.01 & 180.51 & 93.29 & 90.50 & 3.27 \\
\hline $\mathrm{R}_{10}$ & 90.77 & 179.08 & 91.85 & 88.31 & 1.08 \\
\hline $\mathrm{R}_{11}$ & 80.17 & 172.30 & 85.07 & 92.13 & 4.90 \\
\hline $\mathrm{R}_{12}$ & 83.47 & 173.46 & 86.24 & 89.99 & 2.76 \\
\hline $\mathrm{R}_{13}$ & 91.04 & 179.84 & 92.61 & 88.79 & 1.56 \\
\hline $\mathrm{R}_{14}$ & 69.34 & 162.14 & 74.92 & 92.81 & 5.58 \\
\hline $\mathrm{R}_{15}$ & 79.13 & 171.85 & 84.62 & 92.72 & 5.49 \\
\hline $\mathrm{R}_{16}$ & 89.87 & 178.42 & 91.19 & 88.55 & 1.32 \\
\hline $\mathrm{AAD}^{\mathrm{d}}$ & - & - & - & 90.19 & 2.96 \\
\hline
\end{tabular}

(T)/CBS and correction scheme, daverage of absolute difference
反应方法校正即可得到可靠的反应能垒。本文对 23 个目标反应均采用等键反应方法对其能垒进行 了校正, 校正后的反应能垒见表 $\mathrm{S} 2$ 。

\section{3 反应速率常数}

本文使用 Chemrate 程序 ${ }^{28}$ 对所涉及的 25 个反 应计算了其在 $300-1200 \mathrm{~K}$ 范围的速率常数。燃 烧动力学模拟需要的动力学参数通常根据改进的 Arrhenius 方程(见公式(9))进行拟合。

$$
k=A T^{n} \exp (-E / R T)
$$

其中: $A 、 n 、 E$ 为不同温度下反应速率常数对改进 Arrhenius 公式进行拟合得到的动力学参数。对通 过等键反应方法校正后不同温度下速率常数 $k(T)$ 进 行拟合, 得到动力学参数 $(A, n, E)$, 结果列于表 4 中。对甲烷与氢过氧自由基的反应, Aguilera等 ${ }^{9}$ 用 $\operatorname{CCSD}(\mathrm{T}) / \mathrm{cc}-\mathrm{pVTZ}$ 方法计算得到 $700 \mathrm{~K}$ 时速率常 数为 $1.36 \times 10^{5} \mathrm{~cm}^{3} \cdot \mathrm{mol}^{-1} \cdot \mathrm{s}^{-1}$, 而 Carstensen 等 ${ }^{8}$ 用 CBS-QB3 方法所得值为 $6.47 \times 10^{5} \mathrm{~cm}^{3} \cdot \mathrm{mol}^{-1} \cdot \mathrm{s}^{-1}$, Baldwin 等 ${ }^{4}$ 得到的实验值为 $1.37 \times 10^{5} \mathrm{~cm}^{3} \cdot \mathrm{mol}^{-1}$. $\mathrm{s}^{-1}$, 本文通过 $\operatorname{CCSD}(\mathrm{T}) / \mathrm{CBS}$ 方法计算该温度下常 数为 $4.03 \times 10^{4} \mathrm{~cm}^{3} \cdot \mathrm{mol}^{-1} \cdot \mathrm{s}^{-1}$, 与实验值非常接近。

表 4 反应动力学参数 $(A, n, E)$

Table 4 Reaction kinetic parameters $(A, n, E)$

\begin{tabular}{cccc}
\hline Reaction & $A /\left(\mathrm{cm}^{3} \cdot \mathrm{mol}^{-1} \cdot \mathrm{s}^{-1}\right)$ & $n$ & $E /\left(\mathrm{kJ} \cdot \mathrm{mol}^{-1}\right)$ \\
\hline $\mathrm{R}_{1}$ & 1.39 & 3.85 & 87.03 \\
$\mathrm{R}_{2}$ & 1.27 & 3.79 & 69.11 \\
$\mathrm{R}_{3}$ & 1.28 & 3.81 & 65.00 \\
$\mathrm{R}_{4}$ & 1.38 & 3.80 & 55.02 \\
$\mathrm{R}_{5}$ & 0.37 & 3.89 & 64.62 \\
$\mathrm{R}_{6}$ & 0.70 & 3.89 & 58.30 \\
$\mathrm{R}_{7}$ & 0.57 & 3.92 & 64.70 \\
$\mathrm{R}_{8}$ & 0.45 & 3.90 & 46.34 \\
$\mathrm{R}_{9}$ & 0.61 & 3.91 & 65.65 \\
$\mathrm{R}_{10}$ & 0.58 & 3.91 & 64.19 \\
$\mathrm{R}_{11}$ & 0.67 & 3.88 & 58.02 \\
$\mathrm{R}_{12}$ & 0.79 & 3.88 & 59.34 \\
$\mathrm{R}_{13}$ & 0.57 & 3.92 & 64.93 \\
$\mathrm{R}_{14}$ & 0.47 & 3.87 & 48.27 \\
$\mathrm{R}_{15}$ & 0.50 & 3.88 & 57.74 \\
$\mathrm{R}_{16}$ & 0.53 & 3.91 & 63.49 \\
$\mathrm{R}_{17}$ & 0.56 & 3.91 & 64.09 \\
$\mathrm{R}_{18}$ & 0.65 & 3.90 & 54.91 \\
$\mathrm{R}_{19}$ & 0.41 & 3.87 & 49.93 \\
$\mathrm{R}_{20}$ & 0.68 & 3.92 & 64.54 \\
$\mathrm{R}_{21}$ & 0.63 & 3.90 & 54.82 \\
$\mathrm{R}_{22}$ & 0.40 & 3.87 & 47.27 \\
$\mathrm{R}_{23}$ & 0.54 & 3.91 & 63.98 \\
$\mathrm{R}_{24}$ & 0.61 & 3.90 & 54.79 \\
$\mathrm{R}_{25}$ & 0.43 & 3.88 & 46.51 \\
\hline & & & \\
\hline
\end{tabular}



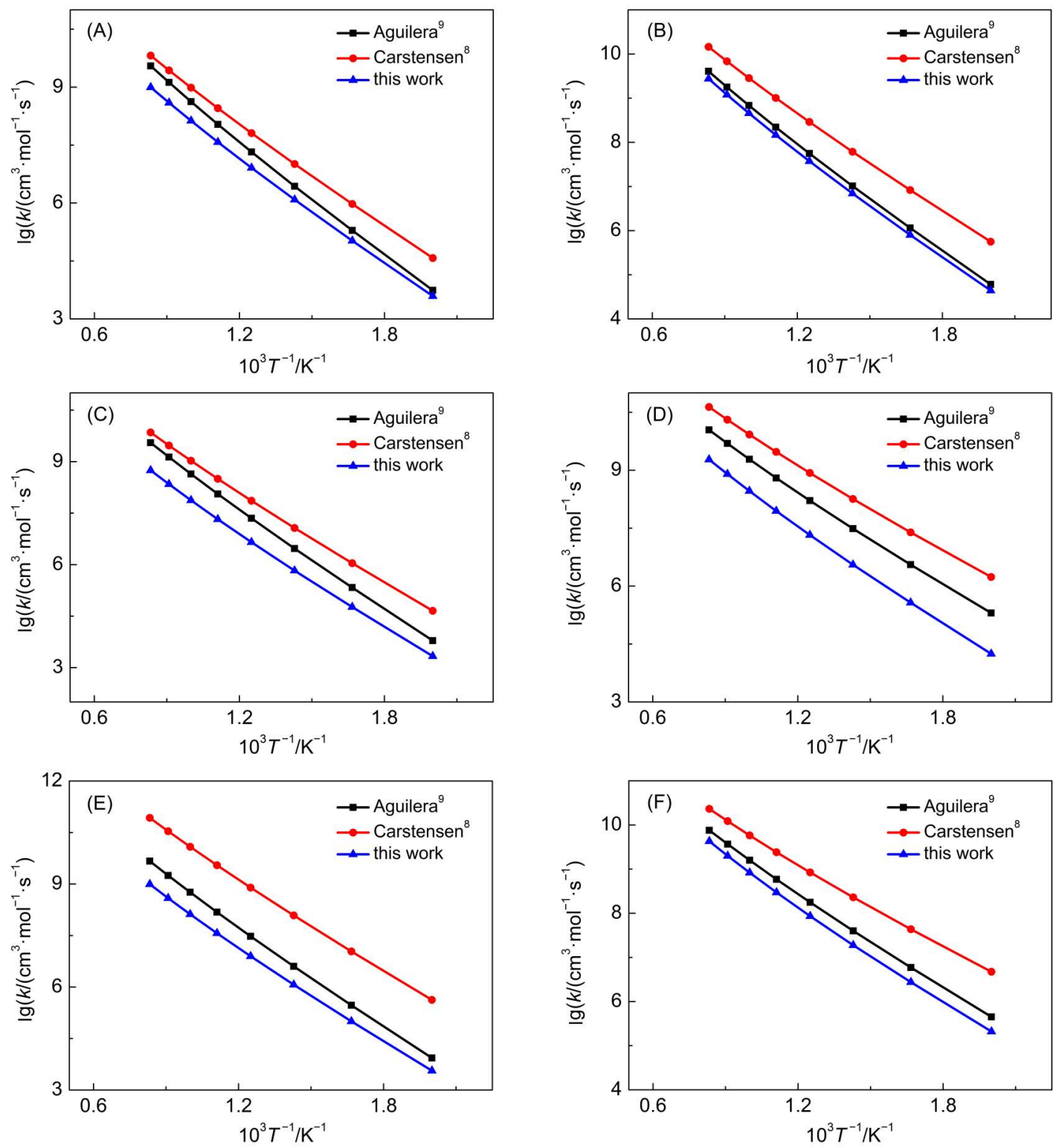

图 2 本文计算的速率常数与文献值比较

Fig.2 Comparison of rate constants calculated in this study with those reported in other studies $k$ : rate constant. (A) $\mathrm{C}_{3} \mathrm{H}_{8}+\mathrm{HO}_{2} \cdot \rightarrow n-\mathrm{C}_{3} \mathrm{H}_{7} \cdot+\mathrm{H}_{2} \mathrm{O}_{2}$; (B) $\mathrm{C}_{3} \mathrm{H}_{8}+\mathrm{HO}_{2} \cdot \rightarrow i-\mathrm{C}_{3} \mathrm{H}_{7} \cdot+\mathrm{H}_{2} \mathrm{O}_{2}$; (C) $n-\mathrm{C}_{4} \mathrm{H}_{10}+\mathrm{HO}_{2} \cdot \rightarrow n-\mathrm{C}_{4} \mathrm{H}_{9} \cdot+\mathrm{H}_{2} \mathrm{O}_{2}$; (D) $n-\mathrm{C}_{4} \mathrm{H}_{10}+\mathrm{HO}_{2} \cdot \rightarrow s-\mathrm{C}_{4} \mathrm{H}_{9} \cdot+\mathrm{H}_{2} \mathrm{O}_{2}$; (E) $i-\mathrm{C}_{4} \mathrm{H}_{10}+\mathrm{HO}_{2} \cdot \rightarrow i-\mathrm{C}_{4} \mathrm{H}_{9} \cdot+\mathrm{H}_{2} \mathrm{O}_{2}$; (F) $i-\mathrm{C}_{4} \mathrm{H}_{10}+\mathrm{HO}_{2} \cdot \rightarrow t-\mathrm{C}_{4} \mathrm{H}_{9} \cdot+\mathrm{H}_{2} \mathrm{O}_{2}$

对乙烷和氢过氧自由基的反应 Aguilera等 ${ }^{9}$ 用 CCSD (T)/cc-pVTZ 方法计算得到 $700 \mathrm{~K}$ 时速率常数为 $3.36 \times 10^{6} \mathrm{~cm}^{3} \cdot \mathrm{mol}^{-1} \cdot \mathrm{s}^{-1}$, 而 Carstensen 等 ${ }^{8}$ 用 CBS$\mathrm{QB} 3$ 方法所得值为 $1.21 \times 10^{8} \mathrm{~m}^{3} \cdot \mathrm{mol}^{-1} \cdot \mathrm{s}^{-1}$, Baldwin 等 ${ }^{3}$ 得到的实验值为 $4.44 \times 10^{6} \mathrm{~cm}^{3} \cdot \mathrm{mol}^{-1} \cdot \mathrm{s}^{-1}$, 本文 通过 $\operatorname{CCSD}(\mathrm{T}) / \mathrm{CBS}$ 方法计算该温度下常数为 $5.52 \times$ $10^{5} \mathrm{~m}^{3} \cdot \mathrm{mol}^{-1} \cdot \mathrm{s}^{-1}$, 与实验值接近。

图 2 中给出了在 500-1200 K 温度范围内本文 用等键反应方法计算的部分反应速率常数和其它 计算值的比较示意图。从图 2 可以看出, 本文用等 键反应方法计算得到的反应 $R_{3} 、 R_{4} 、 R_{5} 、 R_{6} 、 R_{7} 、$
$\mathrm{R}_{8}$ 的速率常数与 Aguilera 等 ${ }^{9}$ 用 $\operatorname{CCSD}(\mathrm{T}) / \mathrm{cc}-\mathrm{pVTZ}$ 方法计算得到的速率常数符合较好, Carstensen等 ${ }^{8}$ 用 CBS-QB3 方法计算得到的速率常数普遍偏高, 因而本文为烷烃分子与氢过氧自由基氢提取这一 类型反应提供了准确可靠的动力学数据。

\section{4 结 论}

采用 $\mathrm{HF} / 6-31+\mathrm{G}(d)$ 方法优化得到了烷烃与氢 过氧自由基氢提取反应的过渡态, 将过渡态理论 与反应类等键反应方法结合, 对 $\mathrm{HF}$ 水平计算得到 的烷烃与氢过氧自由基氢提取反应体系中 23 个反 
应的能垒和速率常数进行了校正, 并选取 $\mathrm{C}_{5}$ 以下 的烷烃为研究对象, 比较了等键反应方法校正后 的反应能垒与高精度 $\mathrm{CCSD}(\mathrm{T}) / \mathrm{CBS}$ 方法计算的反 应能垒, 最大绝对误差为 $5.58 \mathrm{~kJ} \cdot \mathrm{mol}^{-1}$, 在化学反 应精度内。因此, 本文通过在 HF 方法的基础上进 行等键反应方法校正即可得到烷烃与氢过氧自由 基氢提取反应的精确动力学参数, 解决了该类大 分子体系反应的动力学参数的精确计算问题, 对 碳氢化合物的中低温燃烧机理研究具有重要意义。

Supporting Information: Table S1 lists $E_{\infty}^{\mathrm{HF}}$ and $E_{\infty}^{\mathrm{corr}}$ for reactants and transition states involving less than five carbon atoms. Table S2 lists all energy barriers. Optimized geometries of all species involved in this study are also given. This information is available free of charge via the internet at http: //www.whxb.pku. edu.cn.

\section{References}

(1) Battin-Leclerc, F. Prog. Energy Combust. Sci. 2008, 34, 440. doi: 10.1016/j.pecs.2007.10.002

(2) Pousse, E.; Glaude, P. A.; Fournet, R.; Battin-Leclerc, F. Combust. Flame 2009, 156, 954. doi: 10.1016/j. combustflame.2008.09.012

(3) Baldwin, R. R.; Dean, C. E.; Honeyman, M. R.; Walker, R. W. J. Chem. Soc. Faraday Trans. 1986, 1, 89. doi: 10.1039/ F19868200089

(4) Baldwin, R. R.; Jones, P. N.; Walker, R. W. J. Chem. Soc. Faraday Trans. 1988, 2, 199. doi: 10.1039/F29888400199

(5) Handford-Styring, S. M.; Walker, R. W. Phys. Chem. Chem. Phys. 2001, 3, 2043. doi: 10.1039/B101314I

(6) Scott, M.; Walker, R. W. Combust. Flame 2002, 129, 365. doi: 10.1016/S0010-2180(02)00350-4

(7) Baldwin, R. R.; Walker, R. W. Symp. Int. Combust. Proc. 1979, 17, 525. doi: 10.1016/S0082-0784(79)80053-3

(8) Carstensen, H. H.; Dean, A. M.; Deutschmann, O. Proc. Combust. Inst. 2007, 31, 149. doi: 10.1016/j.proci.2006.08.091

(9) Aguilera-Iparraguirre, J.; Curran, H. J.; Klopper, W.; Simmie, J. M. J. Phys. Chem. A 2008, 112, 7047. doi: 10.1021/jp8012464

(10) Truong, T. N. J. Chem. Phys. 2000, 113, 4957. doi: 10.1063/

\subsection{9}

(11) Huynh, L. K.; Ratkiewicz, A.; Truong, T. N. J. Phys. Chem. A 2006, 110, 473. doi: 10.1021/jp051280d

(12) Muszynska, M.; Ratkiewicz, A.; Huynh, L. K.; Truong, T. N. J. Phys. Chem. A 2009, 113, 8327. doi: 10.1021/jp903762x

(13) Bankiewicz, B.; Huynh, L. K.; Ratkiewicz, A.; Truong, T. N. J. Phys. Chem. A 2009, 113, 1564. doi: 10.1021/jp808874j

(14) Zhang, S. W.; Truong, T. N. J. Phys. Chem. A 2003, 107, 1138. doi: 10.1021/jp021265y

(15) Kungwan, N.; Truong, T. N. J. Phys. Chem. A 2005, 109, 7742. doi: $10.1021 /$ jp $051799+$

(16) Huynh, L. K.; Truong, T. N. Theor. Chem. Account 2008, 120 , 107. doi: 10.1007/s00214-007-0311-9

(17) Wang, B. Y.; Tan, L. X.; Yao, Q.; Li, Z. R.; Li, X. Y. Acta Phys. -Chim. Sin. 2012, 28, 2824. [汪必耀, 谈宁馨, 姚 倩, 李泽荣, 李象远. 物理化学学报, 2012, 28, 2824.] doi: 10.3866/ PKU.WHXB201209053

(18) Wang, B. Y.; Li, Z. R.; Tan, N. X.; Yao, Q.; Li, X. Y. J. Phys. Chem. A 2013, 117, 3279. doi: 10.1021/jp400924w

(19) Li, S. J.; Tan, N. X.; Yao, Q.; Li, Z. R.; Li, X. Y. Acta Phys. -Chim. Sin. 2015, 31, 859. [李尚俊, 谈宁馨, 姚 倩, 李泽荣, 李象远. 物理化学学报, 2015, 31, 859.] doi: 10.3866/ PKU.WHXB201503131

(20) Slater, J. C. Phys. Rev. 1951, 81, 385. doi: 10.1103/ PhysRev.81.385

(21) Alecu, I. M.; Zheng, J.; Zhao, Y.; Truhlar, D. G. J. Chem. Theory Comput. 2010, 6, 2872. doi: 10.1021/ct100326h

(22) Truhlar, D. G. Chem. Phys. Lett. 1998, 294, 45. doi: 10.1016/ S0009-2614(98)00866-5

(23) Halkier, A.; Helgaker, T.; Jorgensen, P. J. Chem. Phys. Lett. 1999, 302, 437. doi: 10.1016/S0009-2614(99)00179-7

(24) De Lara-Castells, M. P.; Krems, R. V.; Buchachenko, A. A. J. Chem. Phys. 2001, 115, 10438. doi: 10.1063/1.1415078

(25) Huh, S. B.; Lee, J. S. J. Chem. Phys. 2003, 118, 3035. doi: $10.1063 / 1.1534091$

(26) Hwang, R.; Park, Y. C.; Lee, J. S. Theor. Chem. Acc. 2006, 115, 54. doi: 10.1007/s00214-005-0675-7

(27) Frisch, M. J.; Trucks, G. W.; Schlegel, H. B.; et al. Gaussian 09, Revision A.1; Gaussian Inc: Wallingford, CT, 2009.

(28) Mokrushin, V.; Tsang, W. Chemrate v.1.5.8; National Institute of Standards and Technology: Gaithersburg, MD, 2009. 
Supporting Information for Acta Phys. -Chim. Sin. 2017, 33 (4), 763-768

doi: 10.3866/PKU.WHXB201701091

\title{
烷烃与氢过氧自由基氢提取反应类反应能垒与速率常数的 精确计算
}

\author{
姚 倩 $^{1}$ 彭莉娟 $^{2}$ 李泽荣 ${ }^{1,}$ 李象远 $^{3}$ \\ ( ${ }^{1}$ 四川大学化学学院, 成都 $610064 ;{ }^{2}$ 四川大学空天科学与工程学院, 成都 $610065 ;{ }^{3}$ 四川大学化学工程 \\ 学院, 成都 610065)
}

\section{Accurate Calculation of the Energy Barriers and Rate Constants of Hydrogen Abstraction from Alkanes by Hydroperoxyl Radical}

\author{
YAO Qian ${ }^{1} \quad$ PENG Li-Juan $^{2} \quad$ LI Ze-Rong ${ }^{1, *} \quad$ LI Xiang-Yuan $^{3}$ \\ $\left({ }^{1}\right.$ College of Chemistry, Sichuan University, Chengdu 610064, P. R. China; ${ }^{2}$ School of Aeronautics \& \\ Astronautics, Sichuan University, Chengdu 610065, P. R. China; ${ }^{3}$ School of Chemical Engineering, Sichuan \\ University, Chengdu 610065, P. R. China)
}

${ }^{*}$ Corresponding author. Email: lizerong@ scu.edu.cn; Tel: +86-28-85406139. 
表 $\mathrm{S} 1 \mathrm{C}_{5}$ 以下反应体系反应物和过渡态的 $E_{\infty}^{\mathrm{HF}}$ 和 $E_{\infty}^{\mathrm{corr}}$ 值

Table S1 $E_{\infty}^{\mathrm{HF}}$ and $E_{\infty}^{\mathrm{corr}}$ for reactants and transition states containing less than five carbon atoms

\begin{tabular}{ccc}
\hline & $E_{\infty}^{\mathrm{HF}} /$ Hartree & $E_{\infty}^{\text {corr }} /$ Hartree \\
\hline $\mathrm{CH}_{4}$ & -40.21680 & -0.24564 \\
$\mathrm{HO}_{2}$ & -150.25210 & -0.53658 \\
$\mathrm{TS}_{1}$ & -190.40114 & -0.80874 \\
$\mathrm{C}_{2} \mathrm{H}_{6}$ & -79.26639 & -0.45325 \\
$\mathrm{TS}_{2}$ & -229.45270 & -1.02068 \\
$\mathrm{C}_{3} \mathrm{H}_{8}$ & -118.31741 & -0.66273 \\
$\mathrm{TS}_{3}$ & -268.50354 & -1.23025 \\
$\mathrm{TS}_{4}$ & -268.50576 & -1.23247 \\
$n \mathrm{C}_{4} \mathrm{H}_{10}$ & -157.36832 & -0.87251 \\
$i \mathrm{C}_{4} \mathrm{H}_{10}$ & -157.36881 & -0.87426 \\
$\mathrm{TS}_{5}$ & -307.55447 & -1.44035 \\
$\mathrm{TS}_{6}$ & -307.55548 & -1.44322 \\
$\mathrm{TS}_{7}$ & -307.55478 & -1.44287 \\
$\mathrm{TS}_{8}$ & -307.55966 & -1.44573 \\
$n \mathrm{C}_{5} \mathrm{H}_{12}$ & -196.41914 & -1.08236 \\
$i \mathrm{C}_{5} \mathrm{H}_{12}$ & -196.41768 & -1.08538 \\
$t \mathrm{C}_{5} \mathrm{H}_{12}$ & -196.41948 & -1.08791 \\
$\mathrm{TS}_{9}$ & -346.60510 & -1.65669 \\
$\mathrm{TS}_{10}$ & -346.60533 & -1.65029 \\
$\mathrm{TS}_{11}$ & -346.60631 & -1.65334 \\
$\mathrm{TS}_{12}$ & -346.60595 & -1.65400 \\
$\mathrm{TS}_{13}$ & -346.60358 & -1.65349 \\
$\mathrm{TS}_{14}$ & -346.60777 & -1.65756 \\
$\mathrm{TS}_{15}$ & -346.60495 & -1.65665 \\
$\mathrm{TS}_{16}$ & -346.60398 & -1.65353 \\
\hline & & \\
\hline & &
\end{tabular}

表 S2 HF 方法修正前后反应势垒

Table S2 HF energy barriers $(\Delta V)$ before and after validation

\begin{tabular}{ccc}
\hline \multirow{2}{*}{ Reaction } & \multicolumn{2}{c}{$\Delta V /\left(\mathrm{kJ} \cdot \mathrm{mol}^{-1}\right)$} \\
\cline { 2 - 3 } & $\mathrm{HF}$ & IRM \\
\hline $\mathrm{R}_{1}$ & 191.12 & - \\
$\mathrm{R}_{2}$ & 178.97 & - \\
$\mathrm{R}_{3}$ & 179.66 & 92.43 \\
$\mathrm{R}_{4}$ & 169.73 & 82.50 \\
$\mathrm{R}_{5}$ & 179.31 & 92.08 \\
$\mathrm{R}_{6}$ & 172.64 & 85.42
\end{tabular}




\begin{tabular}{lll}
$\mathrm{R}_{7}$ & 179.63 & 92.41 \\
$\mathrm{R}_{8}$ & 160.93 & 73.70 \\
$\mathrm{R}_{9}$ & 180.51 & 93.29 \\
$\mathrm{R}_{10}$ & 179.08 & 91.85 \\
$\mathrm{R}_{11}$ & 172.30 & 85.07 \\
$\mathrm{R}_{12}$ & 173.46 & 86.24 \\
$\mathrm{R}_{13}$ & 179.84 & 92.61 \\
$\mathrm{R}_{14}$ & 162.14 & 74.92 \\
$\mathrm{R}_{15}$ & 171.85 & 84.62 \\
$\mathrm{R}_{16}$ & 178.42 & 91.19 \\
$\mathrm{R}_{17}$ & 178.99 & 91.76 \\
$\mathrm{R}_{18}$ & 169.72 & 82.49 \\
$\mathrm{R}_{19}$ & 161.53 & 74.30 \\
$\mathrm{R}_{20}$ & 179.49 & 92.26 \\
$\mathrm{R}_{21}$ & 169.63 & 82.40 \\
$\mathrm{R}_{22}$ & 161.38 & 74.15 \\
$\mathrm{R}_{23}$ & 178.86 & 91.63 \\
$\mathrm{R}_{24}$ & 169.59 & 82.36 \\
$\mathrm{R}_{25}$ & 160.69 & 73.46 \\
\hline
\end{tabular}

$\mathrm{HF} / 6-31+\mathrm{G}(d)$ 水平优化得到的几何结构 $\left(\mathrm{TS}_{n}\right.$ 为对应的反应 $\mathrm{R}_{n}$ 的过渡态) $\mathrm{HO}_{2}$.

Cartesian Coordinates $(\AA)$

\begin{tabular}{|c|c|c|c|}
\hline $\mathrm{O}$ & 0.05393700 & -0.60031100 & 0.00000000 \\
\hline $\mathrm{H}$ & -0.86298800 & -0.86127700 & 0.00000000 \\
\hline $\mathrm{O}$ & 0.05393700 & 0.70797100 & 0.00000000 \\
\hline $\mathrm{H}_{2} \mathrm{O}_{2}$ & & & \\
\hline \multicolumn{4}{|c|}{ Cartesian Coordinates $(\AA)$} \\
\hline $\mathrm{O}$ & 0.00000000 & 0.69700700 & -0.05493100 \\
\hline $\mathrm{H}$ & 0.78361200 & 0.90258600 & 0.43944800 \\
\hline $\mathrm{O}$ & 0.00000000 & -0.69700700 & -0.05493100 \\
\hline $\mathrm{H}$ & -0.78361200 & -0.90258600 & 0.43944800 \\
\hline
\end{tabular}

Cartesian Coordinates $(\AA)$ 


\begin{tabular}{rrrr}
\hline $\mathrm{C}$ & 0.00000000 & 0.00000000 & 0.00000000 \\
$\mathrm{H}$ & 0.62584000 & 0.62584000 & 0.62584000 \\
$\mathrm{H}$ & -0.62584000 & -0.62584000 & 0.62584000 \\
$\mathrm{H}$ & -0.62584000 & 0.62584000 & -0.62584000 \\
$\mathrm{H}$ & 0.62584000 & -0.62584000 & -0.62584000
\end{tabular}

\begin{tabular}{ccccc}
\hline $\mathrm{TS}_{1}$ & \multicolumn{4}{c}{ Cartesian Coordinates $(\AA)$} \\
\hline \\
$\mathrm{C}$ & -1.77475800 & -0.15883000 & 0.00405100 \\
$\mathrm{H}$ & -1.94470800 & -0.51279600 & 1.00891500 \\
$\mathrm{H}$ & -1.73194400 & -0.93433000 & -0.74413400 \\
$\mathrm{H}$ & -2.36495700 & 0.70088300 & -0.27214700 \\
$\mathrm{H}$ & -0.50953900 & 0.31623500 & 0.01836700 \\
$\mathrm{O}$ & 0.59949100 & 0.69778900 & 0.01511400 \\
$\mathrm{O}$ & 1.34648500 & -0.44595900 & -0.11588500 \\
$\mathrm{H}$ & 1.63188700 & -0.63165700 & 0.77085400 \\
\hline
\end{tabular}

$\mathrm{CH}_{3}$

Cartesian Coordinates $(\AA)$

\begin{tabular}{rrrr}
\hline $\mathrm{C}$ & 0.00000000 & 0.00000000 & 0.00000000 \\
$\mathrm{H}$ & 0.00000000 & 1.07269900 & 0.00000000 \\
$\mathrm{H}$ & 0.92898400 & -0.53634900 & 0.00000000 \\
$\mathrm{H}$ & -0.92898400 & -0.53634900 & 0.00000000 \\
& & & \\
\hline $\mathrm{C}_{2} \mathrm{H}_{6}$ & \multicolumn{4}{|}{} & \\
& \multicolumn{5}{|}{ Cartesian Coordinates $(\AA)$} \\
$\mathrm{C}$ & 0.00000000 & 0.00000000 & 0.76358900 \\
$\mathrm{H}$ & 0.00000000 & 1.01217200 & 1.15667300 \\
$\mathrm{H}$ & 0.87656700 & -0.50608600 & 1.15667300 \\
$\mathrm{H}$ & -0.87656700 & -0.50608600 & 1.15667300 \\
$\mathrm{C}$ & 0.00000000 & 0.00000000 & -0.76358900 \\
$\mathrm{H}$ & 0.87656700 & 0.50608600 & -1.15667300 \\
$\mathrm{H}$ & 0.00000000 & -1.01217200 & -1.15667300 \\
$\mathrm{H}$ & -0.87656700 & 0.50608600 & -1.15667300 \\
\hline
\end{tabular}

$\mathrm{TS}_{2}$

Cartesian Coordinates $(\AA)$

\begin{tabular}{lrrr}
\hline $\mathrm{C}$ & -1.13762900 & 0.67722800 & 0.32439400 \\
$\mathrm{H}$ & -1.48352400 & 1.59926100 & -0.12247500 \\
$\mathrm{H}$ & -1.02620900 & 0.75845000 & 1.39709600 \\
$\mathrm{H}$ & 0.13105100 & 0.62232900 & -0.09569400 \\
\hline
\end{tabular}




\begin{tabular}{lrrr}
\hline $\mathrm{O}$ & 1.25167700 & 0.50951400 & -0.47482200 \\
$\mathrm{O}$ & 1.72181500 & -0.58669600 & 0.20565000 \\
$\mathrm{H}$ & 2.22024700 & -0.21078800 & 0.92135300 \\
$\mathrm{C}$ & -1.80795100 & -0.58350100 & -0.16902900 \\
$\mathrm{H}$ & -1.79290700 & -0.63698600 & -1.25172900 \\
$\mathrm{H}$ & -1.31538400 & -1.46672000 & 0.22083500 \\
$\mathrm{H}$ & -2.84772700 & -0.61045100 & 0.15180500 \\
\hline
\end{tabular}

$\mathrm{C}_{2} \mathrm{H}_{5}$

Cartesian Coordinates $(\AA)$

\begin{tabular}{crrr}
\hline $\mathrm{C}$ & 0.01415200 & -0.69918800 & 0.00000000 \\
$\mathrm{H}$ & 0.51517200 & -1.09439900 & 0.87822600 \\
$\mathrm{H}$ & -0.99962600 & -1.10191300 & 0.00000000 \\
$\mathrm{H}$ & 0.51517200 & -1.09439900 & -0.87822600 \\
$\mathrm{C}$ & 0.01415200 & 0.79913400 & 0.00000000 \\
$\mathrm{H}$ & -0.10026900 & 1.34551700 & -0.91892400 \\
$\mathrm{H}$ & -0.10026900 & 1.34551700 & 0.91892400 \\
\hline $\mathrm{C}_{3} \mathrm{H}_{8}$ & & & \\
& \multicolumn{3}{c}{ Cartesian Coordinates $(\AA)$} \\
$\mathrm{C}$ & 0.00000000 & 1.27250400 & -0.259868000 \\
$\mathrm{H}$ & -0.87701800 & 1.31715300 & -0.90020800 \\
$\mathrm{H}$ & 0.87701800 & 1.31715300 & -0.90020800 \\
$\mathrm{H}$ & 0.00000000 & 2.16175900 & 0.36351400 \\
$\mathrm{C}$ & 0.00000000 & 0.00000000 & 0.58595100 \\
$\mathrm{H}$ & 0.87009300 & 0.00000000 & 1.23825400 \\
$\mathrm{H}$ & -0.87009300 & 0.00000000 & 1.23825400 \\
$\mathrm{C}$ & 0.00000000 & -1.27250400 & -0.25986800 \\
$\mathrm{H}$ & 0.87701800 & -1.31715300 & -0.90020800 \\
$\mathrm{H}$ & 0.00000000 & -2.16175900 & 0.36351400 \\
$\mathrm{H}$ & -0.87701800 & -1.31715300 & -0.90020800 \\
\hline
\end{tabular}

$\mathrm{TS}_{3}$

Cartesian Coordinates $(\AA)$

\begin{tabular}{rrrr}
\hline $\mathrm{C}$ & -0.41570400 & 0.80940300 & 0.31332800 \\
$\mathrm{H}$ & -0.33232000 & 0.77571500 & 1.39197400 \\
$\mathrm{H}$ & -0.64290600 & 1.80413500 & -0.04757400 \\
$\mathrm{H}$ & 0.85307100 & 0.64818200 & -0.08165800 \\
$\mathrm{O}$ & 1.96757400 & 0.44883000 & -0.43978100 \\
$\mathrm{O}$ & 2.32613500 & -0.70205400 & 0.21815200 \\
$\mathrm{H}$ & 2.83591700 & -0.38992200 & 0.95614700 \\
$\mathrm{C}$ & -1.21239000 & -0.31344400 & -0.31424700 \\
$\mathrm{H}$ & -1.11803700 & -0.26141600 & -1.39454800 \\
$\mathrm{H}$ & -0.79240100 & -1.26650800 & -0.00899700 \\
$\mathrm{C}$ & -2.69457900 & -0.25537600 & 0.07442100 \\
$\mathrm{H}$ & -3.14954200 & 0.67765300 & -0.24543800 \\
$\mathrm{H}$ & -3.24465800 & -1.06917200 & -0.38791000 \\
\hline
\end{tabular}




\begin{tabular}{crrr}
\hline $\mathrm{H}$ & -2.82275500 & -0.33638300 & 1.15002700 \\
\hline$n \mathrm{C}_{3} \mathrm{H}_{7}$ & \multicolumn{3}{c}{ Cartesian Coordinates $(\AA)$} \\
\hline $\mathrm{C}$ & -1.17234000 & -0.41500800 & 0.00000000 \\
$\mathrm{H}$ & -2.12395500 & 0.10902300 & 0.00000000 \\
$\mathrm{H}$ & -1.14184600 & -1.05424100 & 0.87731000 \\
$\mathrm{H}$ & -1.14184600 & -1.05424100 & -0.87731000 \\
$\mathrm{C}$ & 0.00000000 & 0.57948400 & 0.00000000 \\
$\mathrm{H}$ & -0.08463900 & 1.22388300 & -0.87132600 \\
$\mathrm{H}$ & -0.08463900 & 1.22388300 & 0.87132600 \\
$\mathrm{C}$ & 1.34238300 & -0.09167900 & 0.00000000 \\
$\mathrm{H}$ & 1.77833200 & -0.44254600 & -0.91901300 \\
$\mathrm{H}$ & 1.77833200 & -0.44254600 & 0.91901300 \\
\hline
\end{tabular}

$\mathrm{TS}_{4}$

Cartesian Coordinates $(\AA)$

\begin{tabular}{rrrr}
\hline $\mathrm{C}$ & -0.86626200 & 0.04932000 & 0.42281700 \\
$\mathrm{H}$ & -0.74991000 & 0.05839900 & 1.49945700 \\
$\mathrm{H}$ & 0.33786900 & -0.37324400 & 0.05835900 \\
$\mathrm{O}$ & 1.42052800 & -0.76303300 & -0.28215800 \\
$\mathrm{O}$ & 2.28311300 & 0.17991000 & 0.22063600 \\
$\mathrm{H}$ & 2.47871100 & 0.72890200 & -0.52917500 \\
$\mathrm{C}$ & -1.81719600 & -1.01846200 & -0.07307300 \\
$\mathrm{H}$ & -1.79218600 & -1.09494300 & -1.15560400 \\
$\mathrm{H}$ & -1.57241800 & -1.99007400 & 0.34151800 \\
$\mathrm{H}$ & -2.83941600 & -0.78031600 & 0.21766400 \\
$\mathrm{C}$ & -1.02659400 & 1.42939100 & -0.17606000 \\
$\mathrm{H}$ & -1.98976900 & 1.85411300 & 0.10320100 \\
$\mathrm{H}$ & -0.25525300 & 2.10610300 & 0.17526900 \\
$\mathrm{H}$ & -0.98644400 & 1.39455800 & -1.26062100 \\
\hline
\end{tabular}

$i \mathrm{C}_{3} \mathrm{H}_{7}$

Cartesian Coordinates $(\AA)$

\begin{tabular}{rrrr}
\hline $\mathrm{C}$ & -0.01511200 & -0.20123400 & 1.30057100 \\
$\mathrm{H}$ & 0.94392400 & -0.69138700 & 1.48285400 \\
$\mathrm{H}$ & -0.77394200 & -0.97981300 & 1.30686200 \\
$\mathrm{H}$ & -0.20544800 & 0.45900500 & 2.14035900 \\
$\mathrm{C}$ & -0.01511200 & 0.54612100 & 0.00000000 \\
$\mathrm{H}$ & 0.34294300 & 1.56246900 & 0.00000000 \\
$\mathrm{C}$ & -0.01511200 & -0.20123400 & -1.30057100 \\
$\mathrm{H}$ & -0.77394200 & -0.97981300 & -1.30686200 \\
$\mathrm{H}$ & -0.20544800 & 0.45900500 & -2.14035900 \\
$\mathrm{H}$ & 0.94392400 & -0.69138700 & -1.48285400 \\
\hline
\end{tabular}

$n \mathrm{C}_{4} \mathrm{H}_{10}$

Cartesian Coordinates ( $\mathrm{A})$ 


\begin{tabular}{lrrr}
\hline $\mathrm{C}$ & 0.70351400 & 1.82835800 & 0.00000000 \\
$\mathrm{H}$ & 0.19586500 & 2.22074000 & 0.87718500 \\
$\mathrm{H}$ & 1.71537400 & 2.22297600 & 0.00000000 \\
$\mathrm{H}$ & 0.19586500 & 2.22074000 & -0.87718500 \\
$\mathrm{C}$ & 0.70351400 & 0.29999800 & 0.00000000 \\
$\mathrm{H}$ & 1.24772900 & -0.06111000 & -0.87071000 \\
$\mathrm{H}$ & 1.24772900 & -0.06111000 & 0.87071000 \\
$\mathrm{C}$ & -0.70351400 & -0.29999800 & 0.00000000 \\
$\mathrm{H}$ & -1.24772900 & 0.06111000 & -0.87071000 \\
$\mathrm{H}$ & -1.24772900 & 0.06111000 & 0.87071000 \\
$\mathrm{C}$ & -0.70351400 & -1.82835800 & 0.00000000 \\
$\mathrm{H}$ & -0.19586500 & -2.22074000 & 0.87718500 \\
$\mathrm{H}$ & -1.71537400 & -2.22297600 & 0.00000000 \\
$\mathrm{H}$ & -0.19586500 & -2.22074000 & -0.87718500 \\
\hline
\end{tabular}

$\mathrm{TS}_{5}$

Cartesian Coordinates $(\AA)$

\begin{tabular}{|c|c|c|c|}
\hline $\mathrm{C}$ & 0.34498100 & 1.07345600 & 0.17182100 \\
\hline $\mathrm{H}$ & 0.25963700 & 1.99423400 & -0.39042000 \\
\hline $\mathrm{H}$ & 0.49384500 & 1.25302200 & 1.22871700 \\
\hline $\mathrm{H}$ & 1.54169700 & 0.61728200 & -0.21572500 \\
\hline $\mathrm{O}$ & 2.58522700 & 0.16000900 & -0.55249500 \\
\hline $\mathrm{O}$ & 2.79415600 & -0.87299900 & 0.32790100 \\
\hline $\mathrm{H}$ & 3.39173900 & -0.50573800 & 0.96816900 \\
\hline $\mathrm{C}$ & -0.66138100 & -0.00217400 & -0.17275800 \\
\hline $\mathrm{H}$ & -0.63005800 & -0.19485400 & -1.24164900 \\
\hline $\mathrm{H}$ & -0.38034100 & -0.92862900 & 0.31971200 \\
\hline $\mathrm{C}$ & -2.09066700 & 0.38188000 & 0.23463700 \\
\hline $\mathrm{H}$ & -2.36776900 & 1.31353900 & -0.25391400 \\
\hline $\mathrm{H}$ & -2.12129000 & 0.57489200 & 1.30483800 \\
\hline $\mathrm{C}$ & -3.11069100 & -0.70062800 & -0.11711900 \\
\hline $\mathrm{H}$ & -4.11231100 & -0.40638900 & 0.18146300 \\
\hline $\mathrm{H}$ & -3.12641900 & -0.89200100 & -1.18640300 \\
\hline $\mathrm{H}$ & -2.87724200 & -1.63664400 & 0.38247800 \\
\hline \multicolumn{4}{|l|}{$p \mathrm{C}_{4} \mathrm{H}_{9}$} \\
\hline \multicolumn{4}{|c|}{ Cartesian Coordinates $(\AA)$} \\
\hline $\mathrm{C}$ & -1.44758100 & 1.21311900 & 0.00000000 \\
\hline $\mathrm{H}$ & -1.49572700 & 2.29816000 & 0.00000000 \\
\hline $\mathrm{H}$ & -1.98236000 & 0.85860400 & 0.87734400 \\
\hline $\mathrm{H}$ & -1.98236000 & 0.85860400 & -0.87734400 \\
\hline $\mathrm{C}$ & 0.00000000 & 0.72460600 & 0.00000000 \\
\hline $\mathrm{H}$ & 0.51831400 & 1.11998600 & -0.87071300 \\
\hline $\mathrm{H}$ & 0.51831400 & 1.11998600 & 0.87071300 \\
\hline $\mathrm{C}$ & 0.10792300 & -0.81038100 & 0.00000000 \\
\hline $\mathrm{H}$ & -0.41551400 & -1.19828600 & -0.87159600 \\
\hline
\end{tabular}




\begin{tabular}{|c|c|c|c|}
\hline $\mathrm{H}$ & -0.41551400 & -1.19828600 & 0.87159600 \\
\hline $\mathrm{C}$ & 1.52301500 & -1.30878600 & 0.00000000 \\
\hline $\mathrm{H}$ & 2.07735200 & -1.38506000 & 0.91933600 \\
\hline $\mathrm{H}$ & 2.07735200 & -1.38506000 & -0.91933600 \\
\hline \multicolumn{4}{|l|}{$\mathrm{TS}_{6}$} \\
\hline \multicolumn{4}{|c|}{ Cartesian Coordinates $(\AA)$} \\
\hline $\mathrm{C}$ & -0.32805800 & 0.21031800 & 0.34079600 \\
\hline $\mathrm{H}$ & -0.27914300 & 0.16493400 & 1.42276400 \\
\hline $\mathrm{H}$ & 0.83002500 & -0.35498800 & 0.02780100 \\
\hline $\mathrm{O}$ & 1.87156400 & -0.87837900 & -0.26430800 \\
\hline $\mathrm{O}$ & 2.82097800 & -0.05969100 & 0.29623000 \\
\hline $\mathrm{H}$ & 3.12449700 & 0.46608600 & -0.43403600 \\
\hline $\mathrm{C}$ & -1.37025200 & -0.72111100 & -0.25121300 \\
\hline $\mathrm{H}$ & -1.28568100 & -0.71445600 & -1.33502500 \\
\hline $\mathrm{H}$ & -1.15917600 & -1.73654500 & 0.07127100 \\
\hline $\mathrm{C}$ & -2.80466600 & -0.35691100 & 0.15230200 \\
\hline $\mathrm{H}$ & -3.08668500 & 0.62703300 & -0.20841500 \\
\hline $\mathrm{H}$ & -3.50885300 & -1.07257300 & -0.26045800 \\
\hline $\mathrm{H}$ & -2.92109200 & -0.36277700 & 1.23238200 \\
\hline $\mathrm{C}$ & -0.25348400 & 1.62180600 & -0.20151500 \\
\hline $\mathrm{H}$ & -1.13643500 & 2.19330600 & 0.07692700 \\
\hline $\mathrm{H}$ & 0.60910100 & 2.14672000 & 0.19478100 \\
\hline $\mathrm{H}$ & -0.18814500 & 1.62320100 & -1.28558400 \\
\hline
\end{tabular}

${ }_{s} \mathrm{C}_{4} \mathrm{H}_{9}$

Cartesian Coordinates $(\AA)$

\begin{tabular}{crrr}
\hline $\mathrm{C}$ & 1.93588100 & 0.16575300 & -0.08062600 \\
$\mathrm{H}$ & 2.10363300 & 0.91697500 & 0.68614600 \\
$\mathrm{H}$ & 2.74106500 & -0.55962000 & -0.01446600 \\
$\mathrm{H}$ & 2.00614900 & 0.65700400 & -1.04643400 \\
$\mathrm{C}$ & 0.57322700 & -0.50525100 & 0.09558900 \\
$\mathrm{H}$ & 0.44632500 & -1.27788500 & -0.66098100 \\
$\mathrm{H}$ & 0.55470000 & -1.02948200 & 1.05525400 \\
$\mathrm{C}$ & -0.57858500 & 0.45613400 & 0.02136100 \\
$\mathrm{H}$ & -0.42226800 & 1.45792100 & 0.39008800 \\
$\mathrm{C}$ & -1.98812300 & -0.04991800 & -0.06339400 \\
$\mathrm{H}$ & -2.31486000 & -0.49140700 & 0.88072400 \\
$\mathrm{H}$ & -2.68368200 & 0.74726300 & -0.30450400 \\
$\mathrm{H}$ & -2.08545800 & -0.82108000 & -0.82340500 \\
\hline$i \mathrm{C}_{4} \mathrm{H}_{10}$ & & \\
\hline $\mathrm{C}$ & & \\
\hline $\mathrm{C}$ & 0.00000000 & 1.45656300 & -0.09628900 \\
$\mathrm{H}$ & 0.87760000 & 1.98693400 & 0.26254200 \\
$\mathrm{H}$ & 0.00000000 & 1.51481000 & -1.18240400 \\
\hline
\end{tabular}




\begin{tabular}{crrr}
\hline $\mathrm{C}$ & 0.00000000 & 0.00000000 & 0.37374000 \\
$\mathrm{H}$ & 0.00000000 & 0.00000000 & 1.46273000 \\
$\mathrm{C}$ & -1.26142000 & -0.72828100 & -0.09628900 \\
$\mathrm{H}$ & -1.31186400 & -0.75740500 & -1.18240400 \\
$\mathrm{H}$ & -1.28193500 & -1.75349100 & 0.26254200 \\
$\mathrm{H}$ & -2.15953500 & -0.23344300 & 0.26254200 \\
$\mathrm{C}$ & 1.26142000 & -0.72828100 & -0.09628900 \\
$\mathrm{H}$ & 2.15953500 & -0.23344300 & 0.26254200 \\
$\mathrm{H}$ & 1.28193500 & -1.75349100 & 0.26254200 \\
$\mathrm{H}$ & 1.31186400 & -0.75740500 & -1.18240400 \\
\hline
\end{tabular}

$\mathrm{TS}_{7}$

Cartesian Coordinates $(\AA)$

\begin{tabular}{crrr}
\hline $\mathrm{C}$ & -0.15150200 & -0.70604100 & 0.77403100 \\
$\mathrm{H}$ & -0.39791400 & -0.49455700 & 1.80735900 \\
$\mathrm{H}$ & 0.04843800 & -1.75690400 & 0.60893300 \\
$\mathrm{H}$ & 1.07020700 & -0.16968600 & 0.65583600 \\
$\mathrm{O}$ & 2.15416100 & 0.29029200 & 0.49431200 \\
$\mathrm{O}$ & 2.53221400 & -0.17977500 & -0.73978500 \\
$\mathrm{H}$ & 3.09835000 & -0.91482600 & -0.53698800 \\
$\mathrm{C}$ & -1.04156800 & -0.04225500 & -0.25963000 \\
$\mathrm{C}$ & -2.41129200 & -0.73625300 & -0.29735100 \\
$\mathrm{H}$ & -2.92304700 & -0.64377800 & 0.65725100 \\
$\mathrm{H}$ & -3.04541700 & -0.29038200 & -1.05804800 \\
$\mathrm{H}$ & -2.31481200 & -1.79417900 & -0.52325300 \\
$\mathrm{C}$ & -1.18914900 & 1.45739900 & 0.00866500 \\
$\mathrm{H}$ & -1.80692500 & 1.92575000 & -0.75170200 \\
$\mathrm{H}$ & -1.66016000 & 1.63489000 & 0.97281100 \\
$\mathrm{H}$ & -0.22502800 & 1.95382800 & 0.00954800 \\
$\mathrm{H}$ & -0.57362500 & -0.17139200 & -1.23225500 \\
\hline$i \mathrm{C}_{4} \mathrm{H} 9$ & & & \\
\hline & & & \\
$\mathrm{C}$ & $\mathrm{Cartesian} \mathrm{Coordinates}(\AA)$ & \\
$\mathrm{H}$ & 1.05102100 & -0.96609800 & 0.13210600 \\
$\mathrm{H}$ & 2.05154200 & -0.70544300 & -0.19956700 \\
$\mathrm{H}$ & 1.06051100 & -1.00629800 & 1.21806200 \\
$\mathrm{C}$ & 0.82464300 & -1.96252600 & -0.23808900 \\
$\mathrm{H}$ & 0.01432100 & 0.05401700 & -0.37048900 \\
$\mathrm{C}$ & 0.04595000 & 0.03866000 & -1.45886000 \\
$\mathrm{H}$ & 0.35622900 & 1.44716400 & 0.08289000 \\
$\mathrm{H}$ & 1.16752000 & 1.98485400 & -0.37683000 \\
$\mathrm{C}$ & -0.01728700 & 1.82338600 & 1.02025900 \\
$\mathrm{H}$ & -1.39841100 & -0.34204800 & 0.06929300 \\
$\mathrm{H}$ & -2.13906200 & 0.35044100 & -0.31863500 \\
$\mathrm{H}$ & -1.65281100 & -1.33757700 & -0.28276200 \\
& -1.47996400 & -0.34371000 & 1.15362200 \\
\hline & & & \\
\hline
\end{tabular}


$\mathrm{TS}_{8}$

Cartesian Coordinates $(\AA)$

\begin{tabular}{lrrr}
\hline $\mathrm{C}$ & 0.75757500 & -0.03458300 & 0.00033000 \\
$\mathrm{H}$ & -0.47512400 & 0.43501000 & -0.02427900 \\
$\mathrm{O}$ & -1.60645800 & 0.86558500 & -0.04681000 \\
$\mathrm{O}$ & -2.38631700 & -0.25365600 & 0.11337900 \\
$\mathrm{H}$ & -2.66943600 & -0.45965800 & -0.76939500 \\
$\mathrm{C}$ & 0.78892600 & -1.08207900 & -1.09625300 \\
$\mathrm{H}$ & 0.07985500 & -1.87995200 & -0.90178300 \\
$\mathrm{H}$ & 0.56284600 & -0.64934600 & -2.06578400 \\
$\mathrm{H}$ & 1.78008600 & -1.53069000 & -1.15891500 \\
$\mathrm{C}$ & 0.90273200 & -0.59271300 & 1.40309100 \\
$\mathrm{H}$ & 1.89800000 & -1.01670400 & 1.53657000 \\
$\mathrm{H}$ & 0.76801000 & 0.18084600 & 2.15129700 \\
$\mathrm{H}$ & 0.17786500 & -1.37662300 & 1.59259000 \\
$\mathrm{C}$ & 1.60541500 & 1.19209600 & -0.28034000 \\
$\mathrm{H}$ & 1.45020000 & 1.95993800 & 0.47013000 \\
$\mathrm{H}$ & 2.66272300 & 0.92806000 & -0.27127800 \\
$\mathrm{H}$ & 1.37928700 & 1.61735100 & -1.25267700 \\
\hline
\end{tabular}

${ }_{t} \mathrm{C}_{4} \mathrm{H}_{9}$

Cartesian Coordinates $(\AA)$

\begin{tabular}{crrr}
\hline $\mathrm{C}$ & 0.00000000 & 1.48655800 & -0.02286300 \\
$\mathrm{H}$ & 0.00000000 & 1.72683700 & -1.08960300 \\
$\mathrm{H}$ & -0.87877100 & 1.95551800 & 0.40993200 \\
$\mathrm{H}$ & 0.87877100 & 1.95551800 & 0.40993200 \\
$\mathrm{C}$ & 0.00000000 & 0.00000000 & 0.20345800 \\
$\mathrm{C}$ & -1.28739700 & -0.74327900 & -0.02286300 \\
$\mathrm{H}$ & -1.49548400 & -0.86341800 & -1.08960300 \\
$\mathrm{H}$ & -1.25414300 & -1.73879700 & 0.40993200 \\
$\mathrm{H}$ & -2.13291400 & -0.21672100 & 0.40993200 \\
$\mathrm{C}$ & 1.28739700 & -0.74327900 & -0.02286300 \\
$\mathrm{H}$ & 2.13291400 & -0.21672100 & 0.40993200 \\
$\mathrm{H}$ & 1.25414300 & -1.73879700 & 0.40993200 \\
$\mathrm{H}$ & 1.49548400 & -0.86341800 & -1.08960300 \\
\hline$t \mathrm{C}_{5} \mathrm{H}_{12}$ & & & \\
\hline & & & \\
\hline $\mathrm{C}$ & $\mathrm{Cartesian} \mathrm{Coordinates}(\AA)$ & \\
$\mathrm{C}$ & 0.00000000 & 0.00000000 & 0.00000000 \\
$\mathrm{H}$ & 0.88614900 & 0.88614900 & 0.88614900 \\
$\mathrm{H}$ & 1.52648700 & 1.52648700 & 0.28533800 \\
$\mathrm{H}$ & 0.28533800 & 1.52648700 & 1.52648700 \\
$\mathrm{C}$ & 1.52648700 & 0.28533800 & 1.52648700 \\
$\mathrm{H}$ & -0.88614900 & -0.88614900 & 0.88614900 \\
$\mathrm{H}$ & -1.52648700 & -0.28533800 & 1.52648700 \\
& -1.52648700 & -1.52648700 & 0.28533800 \\
\hline
\end{tabular}




\begin{tabular}{|c|c|c|c|}
\hline $\mathrm{H}$ & -0.28533800 & -1.52648700 & 1.52648700 \\
\hline $\mathrm{C}$ & 0.88614900 & -0.88614900 & -0.88614900 \\
\hline $\mathrm{H}$ & 0.28533800 & -1.52648700 & -1.52648700 \\
\hline $\mathrm{H}$ & 1.52648700 & -0.28533800 & -1.52648700 \\
\hline $\mathrm{H}$ & 1.52648700 & -1.52648700 & -0.28533800 \\
\hline $\mathrm{C}$ & -0.88614900 & 0.88614900 & -0.88614900 \\
\hline $\mathrm{H}$ & -1.52648700 & 1.52648700 & -0.28533800 \\
\hline $\mathrm{H}$ & -0.28533800 & 1.52648700 & -1.52648700 \\
\hline $\mathrm{H}$ & -1.52648700 & 0.28533800 & -1.52648700 \\
\hline $\mathrm{TS}_{9}$ & & & \\
\hline \multicolumn{4}{|c|}{ Cartesian Coordinates $(\AA)$} \\
\hline $\mathrm{C}$ & -0.04526200 & -0.14050200 & -1.02579200 \\
\hline $\mathrm{H}$ & 0.04771500 & 0.51076700 & -1.88570600 \\
\hline $\mathrm{H}$ & -0.31803200 & -1.15068700 & -1.30332000 \\
\hline $\mathrm{H}$ & -1.18316200 & 0.29306100 & -0.46279100 \\
\hline $\mathrm{O}$ & -2.20489000 & 0.65611900 & 0.02514400 \\
\hline $\mathrm{O}$ & -2.97603200 & -0.47924700 & 0.07333400 \\
\hline $\mathrm{H}$ & -3.54333600 & -0.39834700 & -0.68400600 \\
\hline $\mathrm{C}$ & 1.07447500 & -0.02498400 & -0.00068600 \\
\hline $\mathrm{C}$ & 0.76264600 & -0.90414400 & 1.21857400 \\
\hline $\mathrm{H}$ & 0.64959900 & -1.94608400 & 0.93194200 \\
\hline $\mathrm{H}$ & 1.56657800 & -0.84635700 & 1.94698100 \\
\hline $\mathrm{H}$ & -0.15485400 & -0.59151600 & 1.70513100 \\
\hline $\mathrm{C}$ & 2.37897600 & -0.50684700 & -0.66242200 \\
\hline $\mathrm{H}$ & 2.62939600 & 0.09791900 & -1.52956400 \\
\hline $\mathrm{H}$ & 3.20749300 & -0.44263400 & 0.03755700 \\
\hline $\mathrm{H}$ & 2.29852400 & -1.54039100 & -0.98727800 \\
\hline $\mathrm{C}$ & 1.23243100 & 1.43838400 & 0.43656100 \\
\hline $\mathrm{H}$ & 0.32922300 & 1.80631200 & 0.91238700 \\
\hline $\mathrm{H}$ & 2.04862000 & 1.54060600 & 1.14598500 \\
\hline $\mathrm{H}$ & 1.45001000 & 2.08094300 & -0.41254700 \\
\hline
\end{tabular}

\begin{tabular}{crrr}
\hline $1-\mathrm{C}_{5} \mathrm{H}_{11}$ & \multicolumn{3}{c}{ Cartesian Coordinates $(\AA)$} \\
\hline $\mathrm{C}$ & -0.02587700 & 0.04106000 & 0.00000000 \\
$\mathrm{C}$ & 1.51654300 & 0.04254600 & 0.00000000 \\
$\mathrm{H}$ & 1.90725100 & 0.54917000 & 0.87776600 \\
$\mathrm{H}$ & 1.90412100 & -0.97330400 & 0.00000000 \\
$\mathrm{H}$ & 1.90725100 & 0.54917000 & -0.87776600 \\
$\mathrm{C}$ & -0.53120200 & -0.68575600 & -1.25572000 \\
$\mathrm{H}$ & -0.17034500 & -1.71030800 & -1.28320700 \\
$\mathrm{H}$ & -1.61655800 & -0.71179700 & -1.28193200 \\
$\mathrm{H}$ & -0.18832900 & -0.18961500 & -2.15965300 \\
$\mathrm{C}$ & -0.53120200 & -0.68575600 & 1.25572000 \\
$\mathrm{H}$ & -0.18832900 & -0.18961500 & 2.15965300 \\
\hline
\end{tabular}




\begin{tabular}{|c|c|c|c|}
\hline $\mathrm{H}$ & -1.61655800 & -0.71179700 & 1.28193200 \\
\hline $\mathrm{H}$ & -0.17034500 & -1.71030800 & 1.28320700 \\
\hline $\mathrm{C}$ & -0.53120200 & 1.46335500 & 0.00000000 \\
\hline $\mathrm{H}$ & -0.57525800 & 2.02285200 & -0.91919900 \\
\hline $\mathrm{H}$ & -0.57525800 & 2.02285200 & 0.91919900 \\
\hline \multicolumn{4}{|l|}{$n \mathrm{C}_{5} \mathrm{H}_{12}$} \\
\hline \multicolumn{4}{|c|}{ Cartesian Coordinates $(\AA)$} \\
\hline $\mathrm{C}$ & 0.00000000 & 2.55077300 & 0.32416500 \\
\hline $\mathrm{H}$ & 0.87715000 & 2.59593000 & 0.96421500 \\
\hline $\mathrm{H}$ & -0.87715000 & 2.59593000 & 0.96421500 \\
\hline $\mathrm{H}$ & 0.00000000 & 3.44015800 & -0.29928400 \\
\hline $\mathrm{C}$ & 0.00000000 & 1.27870400 & -0.52329800 \\
\hline $\mathrm{H}$ & -0.87072900 & 1.28089500 & -1.17623900 \\
\hline $\mathrm{H}$ & 0.87072900 & 1.28089500 & -1.17623900 \\
\hline $\mathrm{C}$ & 0.00000000 & 0.00000000 & 0.31599000 \\
\hline $\mathrm{H}$ & 0.87106900 & 0.00000000 & 0.97015900 \\
\hline $\mathrm{H}$ & -0.87106900 & 0.00000000 & 0.97015900 \\
\hline $\mathrm{C}$ & 0.00000000 & -1.27870400 & -0.52329800 \\
\hline $\mathrm{H}$ & 0.87072900 & -1.28089500 & -1.17623900 \\
\hline $\mathrm{H}$ & -0.87072900 & -1.28089500 & -1.17623900 \\
\hline $\mathrm{C}$ & 0.00000000 & -2.55077300 & 0.32416500 \\
\hline $\mathrm{H}$ & -0.87715000 & -2.59593000 & 0.96421500 \\
\hline $\mathrm{H}$ & 0.00000000 & -3.44015800 & -0.29928400 \\
\hline $\mathrm{H}$ & 0.87715000 & -2.59593000 & 0.96421500 \\
\hline \multicolumn{4}{|l|}{$\mathrm{TS}_{10}$} \\
\hline \multicolumn{4}{|c|}{ Cartesian Coordinates $(\AA)$} \\
\hline $\mathrm{C}$ & 1.00706100 & 1.08090700 & 0.18152200 \\
\hline $\mathrm{H}$ & 0.95741000 & 2.01729200 & -0.35880400 \\
\hline $\mathrm{H}$ & 1.13053900 & 1.23057700 & 1.24631800 \\
\hline $\mathrm{H}$ & 2.20362900 & 0.60820000 & -0.18538100 \\
\hline $\mathrm{O}$ & 3.24620400 & 0.13717400 & -0.50660000 \\
\hline $\mathrm{O}$ & 3.40580700 & -0.92740300 & 0.34612900 \\
\hline $\mathrm{H}$ & 3.99136200 & -0.59399200 & 1.01535400 \\
\hline $\mathrm{C}$ & -0.01320500 & 0.03759300 & -0.21764200 \\
\hline $\mathrm{H}$ & 0.04484700 & -0.12934100 & -1.28965500 \\
\hline $\mathrm{H}$ & 0.23341400 & -0.90648300 & 0.25935700 \\
\hline $\mathrm{C}$ & -1.44367700 & 0.44656700 & 0.15985400 \\
\hline $\mathrm{H}$ & -1.68403400 & 1.39808100 & -0.31162300 \\
\hline $\mathrm{H}$ & -1.49950700 & 0.61409700 & 1.23424900 \\
\hline $\mathrm{C}$ & -2.48675300 & -0.59670300 & -0.24489000 \\
\hline $\mathrm{H}$ & -2.43167300 & -0.76252800 & -1.31874300 \\
\hline $\mathrm{H}$ & -2.24465400 & -1.54811600 & 0.22402200 \\
\hline $\mathrm{C}$ & -3.91110400 & -0.19212400 & 0.13368400 \\
\hline $\mathrm{H}$ & -4.62765700 & -0.95086300 & -0.16625100 \\
\hline
\end{tabular}




\begin{tabular}{crrr}
\hline $\mathrm{H}$ & -4.00777300 & -0.05231400 & 1.20699600 \\
$\mathrm{H}$ & -4.19592800 & 0.73978700 & -0.34724400 \\
\hline $2-\mathrm{C}_{5} \mathrm{H}_{11}$ & \multicolumn{3}{c}{ Cartesian Coordinates $(\AA)$} \\
\hline $\mathrm{C}$ & 2.49848600 & -0.16881100 & 0.00000000 \\
$\mathrm{H}$ & 3.24349400 & -0.95902100 & 0.00000000 \\
$\mathrm{H}$ & 2.67193100 & 0.44881600 & 0.87709600 \\
$\mathrm{H}$ & 2.67193100 & 0.44881600 & -0.87709600 \\
$\mathrm{C}$ & 1.08164700 & -0.74233400 & 0.00000000 \\
$\mathrm{H}$ & 0.95177500 & -1.38220200 & 0.87071800 \\
$\mathrm{H}$ & 0.95177500 & -1.38220200 & -0.87071800 \\
$\mathrm{C}$ & 0.00000000 & 0.33905600 & 0.00000000 \\
$\mathrm{H}$ & 0.12705800 & 0.97918600 & -0.87107800 \\
$\mathrm{H}$ & 0.12705800 & 0.97918600 & 0.87107800 \\
$\mathrm{C}$ & -1.42677400 & -0.23710500 & 0.00000000 \\
$\mathrm{H}$ & -1.54778600 & -0.87642500 & -0.87181400 \\
$\mathrm{H}$ & -1.54778600 & -0.87642500 & 0.87181400 \\
$\mathrm{C}$ & -2.49430900 & 0.81752200 & 0.00000000 \\
$\mathrm{H}$ & -2.80187300 & 1.28515400 & -0.91887900 \\
$\mathrm{H}$ & -2.80187300 & 1.28515400 & 0.91887900 \\
\hline
\end{tabular}

\begin{tabular}{ccrrr}
\hline $\mathrm{TS}_{11}$ & \multicolumn{3}{c}{ Cartesian Coordinates $(\AA)$} \\
\hline $\mathrm{C}$ & -0.28853900 & 0.39745700 & -0.37363500 \\
$\mathrm{H}$ & -0.37651500 & 0.41375400 & -1.45390900 \\
$\mathrm{H}$ & -1.32675000 & -0.36650400 & -0.06441900 \\
$\mathrm{O}$ & -2.25888500 & -1.06916800 & 0.22377900 \\
$\mathrm{O}$ & -3.35178400 & -0.36880400 & -0.22431900 \\
$\mathrm{H}$ & -3.69171000 & 0.04435600 & 0.56028800 \\
$\mathrm{C}$ & 0.91207800 & -0.38962100 & 0.11889900 \\
$\mathrm{H}$ & 0.87574500 & -0.46130800 & 1.20392900 \\
$\mathrm{H}$ & 0.84684700 & -1.40587300 & -0.26150900 \\
$\mathrm{C}$ & 2.25821900 & 0.21422900 & -0.30658400 \\
$\mathrm{H}$ & 2.35412300 & 1.22053700 & 0.09250000 \\
$\mathrm{H}$ & 2.28333300 & 0.30791800 & -1.39024500 \\
$\mathrm{C}$ & -0.56647400 & 1.74032500 & 0.26799600 \\
$\mathrm{H}$ & 0.19810100 & 2.46638100 & -0.00020300 \\
$\mathrm{H}$ & -1.52022500 & 2.13928500 & -0.06047600 \\
$\mathrm{H}$ & -0.58150200 & 1.66246800 & 1.35117400 \\
$\mathrm{C}$ & 3.44646200 & -0.62711300 & 0.15869900 \\
$\mathrm{H}$ & 3.40221800 & -1.63103000 & -0.25406200 \\
$\mathrm{H}$ & 4.38676700 & -0.18215500 & -0.15245900 \\
$\mathrm{H}$ & 3.46443800 & -0.71572700 & 1.24145500 \\
\hline
\end{tabular}

3- $\mathrm{C}_{5} \mathrm{H}_{11}$

Cartesian Coordinates $(\AA)$ 


\begin{tabular}{crrr}
\hline $\mathrm{C}$ & 2.53172300 & -0.29168600 & -0.00408100 \\
$\mathrm{H}$ & 3.40668800 & 0.33701300 & -0.14003900 \\
$\mathrm{H}$ & 2.55767300 & -1.06338200 & -0.76885000 \\
$\mathrm{H}$ & 2.62379100 & -0.78169900 & 0.96158500 \\
$\mathrm{C}$ & 1.24401200 & 0.52655700 & -0.08860400 \\
$\mathrm{H}$ & 1.19573700 & 1.03105200 & -1.05070800 \\
$\mathrm{H}$ & 1.26327500 & 1.30904300 & 0.66724500 \\
$\mathrm{C}$ & -0.01592000 & -0.32264800 & 0.09712300 \\
$\mathrm{H}$ & 0.04356900 & -0.84075600 & 1.05938500 \\
$\mathrm{H}$ & -0.03736400 & -1.10972200 & -0.65614700 \\
$\mathrm{C}$ & -1.29096100 & 0.46792100 & 0.02532600 \\
$\mathrm{H}$ & -1.27781100 & 1.47986000 & 0.39894700 \\
$\mathrm{C}$ & -2.61536200 & -0.23042000 & -0.06532500 \\
$\mathrm{H}$ & -2.87666300 & -0.72225900 & 0.87424200 \\
$\mathrm{H}$ & -3.41629600 & 0.46319600 & -0.29984400 \\
$\mathrm{H}$ & -2.60355100 & -1.00069200 & -0.83245200 \\
\hline
\end{tabular}

\section{$\mathrm{TS}_{12}$}

Cartesian Coordinates $(\AA)$

\begin{tabular}{|c|c|c|c|}
\hline $\mathrm{C}$ & 0.36441900 & 0.05878600 & 0.27123800 \\
\hline $\mathrm{H}$ & 0.32277900 & 0.09350200 & 1.35487700 \\
\hline $\mathrm{H}$ & -0.93257800 & 0.04143600 & -0.01710700 \\
\hline $\mathrm{O}$ & -2.10133400 & 0.01225700 & -0.30262400 \\
\hline $\mathrm{O}$ & -2.56022500 & -1.13668600 & 0.29294500 \\
\hline $\mathrm{H}$ & -2.58403800 & -1.76527500 & -0.41849400 \\
\hline $\mathrm{C}$ & 0.88011400 & 1.34234300 & -0.35245600 \\
\hline $\mathrm{C}$ & 0.90618700 & -1.25609400 & -0.25969700 \\
\hline $\mathrm{H}$ & 0.28138000 & -2.06640900 & 0.10511800 \\
\hline $\mathrm{H}$ & 0.83404400 & -1.26570700 & -1.34468400 \\
\hline $\mathrm{C}$ & 2.35695000 & -1.52865800 & 0.15844600 \\
\hline $\mathrm{H}$ & 2.68131500 & -2.49884100 & -0.20517500 \\
\hline $\mathrm{H}$ & 3.03815700 & -0.78407800 & -0.23977000 \\
\hline $\mathrm{H}$ & 2.45800700 & -1.52967200 & 1.23999300 \\
\hline $\mathrm{C}$ & 0.21701000 & 2.60038300 & 0.20954100 \\
\hline $\mathrm{H}$ & -0.85380100 & 2.59298700 & 0.03723100 \\
\hline $\mathrm{H}$ & 0.38049600 & 2.68228500 & 1.28041800 \\
\hline $\mathrm{H}$ & 0.62576100 & 3.49151500 & -0.25654000 \\
\hline $\mathrm{H}$ & 1.95588600 & 1.40433500 & -0.19119400 \\
\hline $\mathrm{H}$ & 0.73697100 & 1.29880100 & -1.42966900 \\
\hline \multicolumn{4}{|l|}{$4-\mathrm{C}_{5} \mathrm{H}_{11}$} \\
\hline \multicolumn{4}{|c|}{ Cartesian Coordinates $(\AA)$} \\
\hline $\mathrm{C}$ & 0.04278400 & -0.41089200 & 2.51467800 \\
\hline $\mathrm{H}$ & 0.83704300 & -1.14729400 & 2.43724900 \\
\hline $\mathrm{H}$ & -0.89908600 & -0.94665200 & 2.59396300 \\
\hline $\mathrm{H}$ & 0.18739100 & 0.14177000 & 3.43807400 \\
\hline
\end{tabular}




\begin{tabular}{|c|c|c|c|}
\hline $\mathrm{C}$ & 0.04278400 & 0.52575700 & 1.30573300 \\
\hline $\mathrm{H}$ & -0.73647700 & 1.28112700 & 1.44013000 \\
\hline $\mathrm{H}$ & 0.98085300 & 1.07731000 & 1.27214800 \\
\hline $\mathrm{C}$ & -0.16208300 & -0.18783700 & 0.00000000 \\
\hline $\mathrm{H}$ & -0.79377000 & -1.06387300 & 0.00000000 \\
\hline $\mathrm{C}$ & 0.04278400 & 0.52575700 & -1.30573300 \\
\hline $\mathrm{H}$ & 0.98085300 & 1.07731000 & -1.27214800 \\
\hline $\mathrm{H}$ & -0.73647700 & 1.28112700 & -1.44013000 \\
\hline $\mathrm{C}$ & 0.04278400 & -0.41089200 & -2.51467800 \\
\hline $\mathrm{H}$ & -0.89908600 & -0.94665200 & -2.59396300 \\
\hline $\mathrm{H}$ & 0.18739100 & 0.14177000 & -3.43807400 \\
\hline $\mathrm{H}$ & 0.83704300 & -1.14729400 & -2.43724900 \\
\hline$i \mathrm{C}_{5} \mathrm{H}_{12}$ & & & \\
\hline \multicolumn{4}{|c|}{ Cartesian Coordinates $(\AA)$} \\
\hline $\mathrm{C}$ & 2.09635600 & -0.02956400 & -0.11177800 \\
\hline $\mathrm{H}$ & 2.19529900 & 0.98473800 & 0.26014600 \\
\hline $\mathrm{H}$ & 2.20857300 & 0.00072100 & -1.19267300 \\
\hline $\mathrm{H}$ & 2.92685200 & -0.60383700 & 0.28828300 \\
\hline $\mathrm{C}$ & 0.76196100 & -0.66261100 & 0.28676500 \\
\hline $\mathrm{H}$ & 0.77596900 & -1.71049700 & -0.00550300 \\
\hline $\mathrm{H}$ & 0.66708100 & -0.65235700 & 1.37193300 \\
\hline $\mathrm{C}$ & -0.48040700 & -0.00281700 & -0.33096100 \\
\hline $\mathrm{H}$ & -0.35255800 & -0.00279300 & -1.41349700 \\
\hline $\mathrm{C}$ & -1.73494900 & -0.82054800 & -0.00865600 \\
\hline $\mathrm{H}$ & -1.91180500 & -0.85566300 & 1.06399400 \\
\hline $\mathrm{H}$ & -2.61677500 & -0.38873400 & -0.47353300 \\
\hline $\mathrm{H}$ & -1.64199100 & -1.84376800 & -0.36162900 \\
\hline $\mathrm{C}$ & -0.66104400 & 1.44979800 & 0.12178100 \\
\hline $\mathrm{H}$ & -0.75613700 & 1.50955700 & 1.20393500 \\
\hline $\mathrm{H}$ & 0.17352300 & 2.07678800 & -0.17307400 \\
\hline $\mathrm{H}$ & -1.55953000 & 1.88029800 & -0.31128500 \\
\hline
\end{tabular}

$\mathrm{TS}_{13}$

Cartesian Coordinates $(\AA)$

\begin{tabular}{lrrr}
\hline $\mathrm{C}$ & -0.12285800 & -0.69169100 & 0.41496800 \\
$\mathrm{H}$ & 0.15363500 & -1.72054400 & 0.23350200 \\
$\mathrm{H}$ & -0.34302700 & -0.50224800 & 1.45848500 \\
$\mathrm{H}$ & -1.34125200 & -0.64235000 & -0.13852400 \\
$\mathrm{O}$ & -2.42020500 & -0.60678200 & -0.63873900 \\
$\mathrm{O}$ & -3.21990800 & -0.04074300 & 0.32337500 \\
$\mathrm{H}$ & -3.65126800 & -0.78554200 & 0.72475700 \\
$\mathrm{C}$ & 0.72815100 & 0.36308800 & -0.26927600 \\
$\mathrm{H}$ & 0.72860300 & 0.15069200 & -1.33699800 \\
$\mathrm{C}$ & 0.13799700 & 1.76085000 & -0.05856000 \\
$\mathrm{H}$ & 0.13365800 & 2.02487700 & 0.99625100 \\
\hline
\end{tabular}




\begin{tabular}{|c|c|c|c|}
\hline $\mathrm{H}$ & 0.72323500 & 2.50920900 & -0.58452300 \\
\hline $\mathrm{H}$ & -0.88257600 & 1.81760100 & -0.41924500 \\
\hline $\mathrm{C}$ & 2.18995300 & 0.31316700 & 0.21857900 \\
\hline $\mathrm{H}$ & 2.71928600 & 1.15049400 & -0.22991400 \\
\hline $\mathrm{H}$ & 2.21320000 & 0.48043300 & 1.29411200 \\
\hline $\mathrm{C}$ & 2.94079500 & -0.97650200 & -0.11639100 \\
\hline $\mathrm{H}$ & 3.98336900 & -0.89752600 & 0.17643800 \\
\hline $\mathrm{H}$ & 2.52601800 & -1.83826400 & 0.39630400 \\
\hline $\mathrm{H}$ & 2.91380300 & -1.18010400 & -1.18363800 \\
\hline $5-\mathrm{C}_{5} \mathrm{H}_{11}$ & & & \\
\hline \multicolumn{4}{|c|}{ Cartesian Coordinates $(\AA)$} \\
\hline $\mathrm{C}$ & 1.79078200 & -0.70727100 & -0.02197400 \\
\hline $\mathrm{H}$ & 1.73258900 & -1.73292900 & -0.37482700 \\
\hline $\mathrm{H}$ & 1.97605000 & -0.73541700 & 1.04911400 \\
\hline $\mathrm{H}$ & 2.64823300 & -0.23799800 & -0.49400400 \\
\hline $\mathrm{C}$ & 0.49918800 & 0.05887600 & -0.33331500 \\
\hline $\mathrm{H}$ & 0.35809600 & 0.05594700 & -1.41335000 \\
\hline $\mathrm{C}$ & -0.71554500 & -0.65989100 & 0.29116000 \\
\hline $\mathrm{H}$ & -0.61007200 & -0.65677900 & 1.37453600 \\
\hline $\mathrm{H}$ & -0.69211100 & -1.70323300 & -0.01673900 \\
\hline $\mathrm{C}$ & -2.06790900 & -0.05956800 & -0.09229800 \\
\hline $\mathrm{H}$ & -2.20225800 & -0.05351200 & -1.17107800 \\
\hline $\mathrm{H}$ & -2.88167800 & -0.63686500 & 0.33666900 \\
\hline $\mathrm{H}$ & -2.16870700 & 0.96176800 & 0.26026300 \\
\hline $\mathrm{C}$ & 0.61144500 & 1.49066200 & 0.11738100 \\
\hline $\mathrm{H}$ & 0.09548700 & 2.28177500 & -0.39651600 \\
\hline $\mathrm{H}$ & 1.03660500 & 1.72040200 & 1.08020700 \\
\hline \multicolumn{4}{|l|}{$\mathrm{TS}_{14}$} \\
\hline \multicolumn{4}{|c|}{ Cartesian Coordinates $(\AA)$} \\
\hline $\mathrm{C}$ & -0.32179600 & 0.40596300 & 0.05310900 \\
\hline $\mathrm{H}$ & 0.93428600 & 0.27121200 & -0.31751600 \\
\hline $\mathrm{O}$ & 2.08937500 & 0.14670800 & -0.67361900 \\
\hline $\mathrm{O}$ & 2.56705100 & -0.87659700 & 0.10758400 \\
\hline $\mathrm{H}$ & 3.04008800 & -0.43069200 & 0.79973400 \\
\hline $\mathrm{C}$ & -0.24086200 & 0.61034900 & 1.55546000 \\
\hline $\mathrm{H}$ & 0.15810300 & -0.26705300 & 2.05399000 \\
\hline $\mathrm{H}$ & 0.38983800 & 1.45954000 & 1.80018500 \\
\hline $\mathrm{H}$ & -1.22630300 & 0.80957900 & 1.97259800 \\
\hline $\mathrm{C}$ & -0.76595900 & 1.63767900 & -0.71659600 \\
\hline $\mathrm{H}$ & -0.14170600 & 2.49288100 & -0.47848500 \\
\hline $\mathrm{H}$ & -0.71803100 & 1.47308800 & -1.78799500 \\
\hline $\mathrm{H}$ & -1.79132800 & 1.90250000 & -0.46481700 \\
\hline $\mathrm{C}$ & -0.94028800 & -0.91196800 & -0.39868000 \\
\hline $\mathrm{H}$ & -0.78553600 & -1.02075300 & -1.46836000 \\
\hline
\end{tabular}




\begin{tabular}{|c|c|c|c|}
\hline $\mathrm{H}$ & -0.40439900 & -1.72944400 & 0.07444900 \\
\hline $\mathrm{C}$ & -2.43803800 & -1.04891300 & -0.09302800 \\
\hline $\mathrm{H}$ & -3.02217800 & -0.27540500 & -0.58151400 \\
\hline $\mathrm{H}$ & -2.80377000 & -2.00741500 & -0.44800000 \\
\hline $\mathrm{H}$ & -2.63880500 & -0.99758900 & 0.97242900 \\
\hline $6-\mathrm{C}_{5} \mathrm{H}_{11}$ & & & \\
\hline \multicolumn{4}{|c|}{ Cartesian Coordinates $(\AA)$} \\
\hline $\mathrm{C}$ & 2.06967500 & -0.03478500 & -0.18273900 \\
\hline $\mathrm{H}$ & 2.08466000 & 0.07999800 & -1.26274500 \\
\hline $\mathrm{H}$ & 2.20948700 & 0.94610700 & 0.25904800 \\
\hline $\mathrm{H}$ & 2.92428500 & -0.64243900 & 0.09939300 \\
\hline $\mathrm{C}$ & 0.76912800 & -0.69447000 & 0.28401600 \\
\hline $\mathrm{H}$ & 0.78575800 & -0.76823900 & 1.37642100 \\
\hline $\mathrm{H}$ & 0.74752500 & -1.71949500 & -0.07761700 \\
\hline $\mathrm{C}$ & -0.50185000 & -0.00278400 & -0.14757100 \\
\hline $\mathrm{C}$ & -0.63854200 & 1.48054300 & 0.06905600 \\
\hline $\mathrm{H}$ & 0.20914200 & 2.03402800 & -0.31996300 \\
\hline $\mathrm{H}$ & -1.53235200 & 1.86541500 & -0.41193800 \\
\hline $\mathrm{H}$ & -0.71719900 & 1.71978700 & 1.13337800 \\
\hline $\mathrm{C}$ & -1.76609000 & -0.81791400 & -0.10946700 \\
\hline $\mathrm{H}$ & -1.61312200 & -1.81265700 & -0.51727200 \\
\hline $\mathrm{H}$ & -2.12851600 & -0.94296300 & 0.91477600 \\
\hline $\mathrm{H}$ & -2.56358800 & -0.34308400 & -0.67325000 \\
\hline
\end{tabular}

$\mathrm{TS}_{15}$

Cartesian Coordinates $(\AA)$

\begin{tabular}{lrrr}
\hline $\mathrm{C}$ & 0.14033800 & 0.67945100 & -0.45646900 \\
$\mathrm{H}$ & -0.03811100 & 0.81707000 & -1.51768700 \\
$\mathrm{H}$ & -1.09502300 & 0.44939800 & -0.03067500 \\
$\mathrm{O}$ & -2.21501100 & 0.23438600 & 0.35438300 \\
$\mathrm{O}$ & -2.62403200 & -0.83455900 & -0.40529100 \\
$\mathrm{H}$ & -3.14814100 & -0.43724100 & -1.09027700 \\
$\mathrm{C}$ & 0.90919600 & -0.60727800 & -0.17230000 \\
$\mathrm{H}$ & 0.33554600 & -1.41303200 & -0.62320400 \\
$\mathrm{C}$ & 0.54566900 & 1.96642000 & 0.23455300 \\
$\mathrm{H}$ & 1.55604800 & 2.25746600 & -0.04596100 \\
$\mathrm{H}$ & -0.11741400 & 2.77671300 & -0.04901800 \\
$\mathrm{H}$ & 0.51479200 & 1.87349500 & 1.31382300 \\
$\mathrm{C}$ & 2.28542400 & -0.58481900 & -0.85600400 \\
$\mathrm{H}$ & 2.92767900 & 0.18414700 & -0.43641200 \\
$\mathrm{H}$ & 2.78850900 & -1.53843900 & -0.72560700 \\
$\mathrm{H}$ & 2.19663700 & -0.40114300 & -1.92305800 \\
$\mathrm{C}$ & 1.04360200 & -0.91453200 & 1.32217400 \\
$\mathrm{H}$ & 0.07675000 & -0.91559700 & 1.81397500 \\
$\mathrm{H}$ & 1.48970800 & -1.89437400 & 1.46400000 \\
\hline
\end{tabular}




\begin{tabular}{crrr}
\hline $\mathrm{H}$ & 1.67998600 & -0.19253900 & 1.82563500 \\
\hline $7-\mathrm{C}_{5} \mathrm{H}_{11}$ & \multicolumn{3}{c}{ Cartesian Coordinates $(\AA)$} \\
\hline $\mathrm{C}$ & -1.64436400 & -0.94585400 & -0.03091900 \\
$\mathrm{H}$ & -1.44416900 & -1.95545500 & -0.37618500 \\
$\mathrm{H}$ & -1.85251600 & -0.99451000 & 1.03526800 \\
$\mathrm{H}$ & -2.54431100 & -0.59328400 & -0.52686200 \\
$\mathrm{C}$ & -0.45851400 & -0.01821000 & -0.31443900 \\
$\mathrm{H}$ & -0.29365100 & -0.00215300 & -1.39149700 \\
$\mathrm{C}$ & 0.80015500 & -0.52931300 & 0.33848600 \\
$\mathrm{H}$ & 0.71480900 & -0.92151400 & 1.34094900 \\
$\mathrm{C}$ & 2.15880900 & -0.09122900 & -0.12427900 \\
$\mathrm{H}$ & 2.40831800 & 0.91188700 & 0.22679500 \\
$\mathrm{H}$ & 2.93308100 & -0.75783100 & 0.24256900 \\
$\mathrm{H}$ & 2.21940000 & -0.07347400 & -1.20938600 \\
$\mathrm{C}$ & -0.78046300 & 1.42218600 & 0.12325200 \\
$\mathrm{H}$ & -0.96211800 & 1.46985900 & 1.19399900 \\
$\mathrm{H}$ & 0.03633100 & 2.09966400 & -0.10609300 \\
$\mathrm{H}$ & -1.66890800 & 1.79132900 & -0.38216500 \\
\hline
\end{tabular}

\begin{tabular}{|c|c|c|c|}
\hline \multicolumn{4}{|c|}{ Cartesian Coordinates $(\AA)$} \\
\hline $\mathrm{C}$ & -0.60716500 & -0.93607500 & 0.31615400 \\
\hline $\mathrm{H}$ & -0.44332300 & -1.91823700 & -0.10427100 \\
\hline $\mathrm{H}$ & -0.74151300 & -0.96763100 & 1.38996600 \\
\hline $\mathrm{H}$ & -1.84056500 & -0.65797300 & -0.11895000 \\
\hline $\mathrm{O}$ & -2.92473200 & -0.35725800 & -0.50466500 \\
\hline $\mathrm{O}$ & -3.22551200 & 0.77953500 & 0.20430300 \\
\hline $\mathrm{H}$ & -3.78242700 & 0.46621200 & 0.90688700 \\
\hline $\mathrm{C}$ & 0.28034600 & 0.17834000 & -0.19594800 \\
\hline $\mathrm{H}$ & 0.29641100 & 0.15457300 & -1.28296500 \\
\hline $\mathrm{H}$ & -0.16124200 & 1.12928500 & 0.08707200 \\
\hline $\mathrm{C}$ & 1.72671900 & 0.12663700 & 0.33439600 \\
\hline $\mathrm{H}$ & 1.68272300 & 0.13367200 & 1.42307200 \\
\hline $\mathrm{C}$ & 2.49371900 & 1.37457200 & -0.11237500 \\
\hline $\mathrm{H}$ & 3.50428900 & 1.37560600 & 0.28585000 \\
\hline $\mathrm{H}$ & 2.56584800 & 1.41986500 & -1.19637400 \\
\hline $\mathrm{H}$ & 2.00299300 & 2.28281500 & 0.22485200 \\
\hline $\mathrm{C}$ & 2.46369700 & -1.14308200 & -0.10256900 \\
\hline $\mathrm{H}$ & 1.98533100 & -2.04241800 & 0.27179200 \\
\hline $\mathrm{H}$ & 2.50391100 & -1.21453200 & -1.18715400 \\
\hline $\mathrm{H}$ & 3.48561600 & -1.14179700 & 0.26516400 \\
\hline
\end{tabular}

8- $\mathrm{C}_{5} \mathrm{H}_{11}$

Cartesian Coordinates $(\AA)$ 


\begin{tabular}{crrr}
\hline $\mathrm{C}$ & -2.12589900 & -0.09040400 & -0.10940600 \\
$\mathrm{H}$ & -2.50376200 & -0.24661400 & -1.10506000 \\
$\mathrm{H}$ & -2.64483700 & 0.61675300 & 0.51186200 \\
$\mathrm{C}$ & -0.80719800 & -0.67889700 & 0.29986300 \\
$\mathrm{H}$ & -0.70866900 & -0.63849700 & 1.38274500 \\
$\mathrm{H}$ & -0.79150500 & -1.73313000 & 0.02847900 \\
$\mathrm{C}$ & 0.42581300 & -0.00052600 & -0.33609900 \\
$\mathrm{H}$ & 0.29398600 & -0.02285100 & -1.41714300 \\
$\mathrm{C}$ & 1.69804800 & -0.78244000 & 0.00103900 \\
$\mathrm{H}$ & 1.63312800 & -1.81167000 & -0.34052500 \\
$\mathrm{H}$ & 2.57002300 & -0.33307400 & -0.46572500 \\
$\mathrm{H}$ & 1.87160100 & -0.80028300 & 1.07460400 \\
$\mathrm{C}$ & 0.55792300 & 1.46233200 & 0.09552900 \\
$\mathrm{H}$ & -0.31811700 & 2.04171100 & -0.17691800 \\
$\mathrm{H}$ & 0.68528600 & 1.53807800 & 1.17334000 \\
$\mathrm{H}$ & 1.42074100 & 1.92919100 & -0.37120600 \\
\hline
\end{tabular}

$n \mathrm{C}_{6} \mathrm{H}_{14}$

Cartesian Coordinates $(\AA)$

\begin{tabular}{|c|c|c|c|}
\hline $\mathrm{C}$ & -1.41219400 & 2.89190400 & 0.00000000 \\
\hline $\mathrm{H}$ & -0.90452900 & 3.28440400 & 0.87704600 \\
\hline $\mathrm{H}$ & -2.42402300 & 3.28671600 & 0.00000000 \\
\hline $\mathrm{H}$ & -0.90452900 & 3.28440400 & -0.87704600 \\
\hline $\mathrm{C}$ & -1.41219400 & 1.36360200 & 0.00000000 \\
\hline $\mathrm{H}$ & -1.95709500 & 1.00354000 & 0.87050900 \\
\hline $\mathrm{H}$ & -1.95709500 & 1.00354000 & -0.87050900 \\
\hline $\mathrm{C}$ & -0.00471600 & 0.76472400 & 0.00000000 \\
\hline $\mathrm{H}$ & 0.53912400 & 1.12836600 & 0.87087400 \\
\hline $\mathrm{H}$ & 0.53912400 & 1.12836600 & -0.87087400 \\
\hline $\mathrm{C}$ & 0.00471600 & -0.76472400 & 0.00000000 \\
\hline $\mathrm{H}$ & -0.53912400 & -1.12836600 & -0.87087400 \\
\hline $\mathrm{H}$ & -0.53912400 & -1.12836600 & 0.87087400 \\
\hline $\mathrm{C}$ & 1.41219400 & -1.36360200 & 0.00000000 \\
\hline $\mathrm{H}$ & 1.95709500 & -1.00354000 & 0.87050900 \\
\hline $\mathrm{H}$ & 1.95709500 & -1.00354000 & -0.87050900 \\
\hline $\mathrm{C}$ & 1.41219400 & -2.89190400 & 0.00000000 \\
\hline $\mathrm{H}$ & 0.90452900 & -3.28440400 & -0.87704600 \\
\hline $\mathrm{H}$ & 2.42402300 & -3.28671600 & 0.00000000 \\
\hline $\mathrm{H}$ & 0.90452900 & -3.28440400 & 0.87704600 \\
\hline $\mathrm{TS}_{17}$ & & & \\
\hline \multicolumn{4}{|c|}{ Cartesian Coordinates $(\AA)$} \\
\hline $\mathrm{C}$ & -4.42759600 & -0.70547100 & -0.09734800 \\
\hline $\mathrm{H}$ & -4.45652800 & -0.94256200 & -1.15742500 \\
\hline $\mathrm{H}$ & -5.39329200 & -0.28972900 & 0.17460700 \\
\hline $\mathrm{H}$ & -4.30475900 & -1.63873200 & 0.44556600 \\
\hline
\end{tabular}




\begin{tabular}{|c|c|c|c|}
\hline $\mathrm{C}$ & -3.29507500 & 0.27079100 & 0.21943900 \\
\hline $\mathrm{H}$ & -3.46575500 & 1.20532000 & -0.31149000 \\
\hline $\mathrm{H}$ & -3.31527900 & 0.51420700 & 1.27996700 \\
\hline $\mathrm{C}$ & -1.91491900 & -0.27645200 & -0.14858300 \\
\hline $\mathrm{H}$ & -1.89662700 & -0.52073600 & -1.20955100 \\
\hline $\mathrm{H}$ & -1.74621700 & -1.21201300 & 0.38238100 \\
\hline $\mathrm{C}$ & -0.77626400 & 0.69545700 & 0.16584200 \\
\hline $\mathrm{H}$ & -0.79334600 & 0.94166600 & 1.22620800 \\
\hline $\mathrm{H}$ & -0.94051100 & 1.62955100 & -0.36869300 \\
\hline $\mathrm{C}$ & 0.60427400 & 0.13449100 & -0.20281100 \\
\hline $\mathrm{H}$ & 0.62413000 & -0.11268400 & -1.26060900 \\
\hline $\mathrm{H}$ & 0.77726500 & -0.79152200 & 0.33770400 \\
\hline $\mathrm{C}$ & 1.72070400 & 1.10836900 & 0.10369800 \\
\hline $\mathrm{H}$ & 1.88024900 & 1.32138000 & 1.15279800 \\
\hline $\mathrm{H}$ & 2.86302700 & 0.50654100 & -0.24638700 \\
\hline $\mathrm{H}$ & 1.74132300 & 2.00498900 & -0.50203500 \\
\hline $\mathrm{O}$ & 3.85660900 & -0.07467900 & -0.54165300 \\
\hline $\mathrm{O}$ & 3.94259100 & -1.07945800 & 0.39053600 \\
\hline $\mathrm{H}$ & 4.56597700 & -0.74569100 & 1.02447200 \\
\hline$p \mathrm{C}_{6} \mathrm{H}_{13}$ & & & \\
\hline \multicolumn{4}{|c|}{ Cartesian Coordinates $(\AA)$} \\
\hline $\mathrm{C}$ & -3.13859700 & 0.20940600 & -0.00009000 \\
\hline $\mathrm{H}$ & -3.22545400 & 0.84582000 & 0.87652200 \\
\hline $\mathrm{H}$ & -3.98534100 & -0.47071800 & 0.00048000 \\
\hline $\mathrm{H}$ & -3.22549400 & 0.84439800 & -0.87772800 \\
\hline $\mathrm{C}$ & -1.81416100 & -0.55358000 & 0.00049600 \\
\hline $\mathrm{H}$ & -1.77370800 & -1.20449400 & 0.87168900 \\
\hline $\mathrm{H}$ & -1.77377500 & -1.20596900 & -0.86960000 \\
\hline $\mathrm{C}$ & -0.59299200 & 0.36770900 & -0.00032600 \\
\hline $\mathrm{H}$ & -0.63632100 & 1.02091200 & 0.87024400 \\
\hline $\mathrm{H}$ & -0.63646700 & 1.01950500 & -0.87194500 \\
\hline $\mathrm{C}$ & 0.73606600 & -0.38893300 & 0.00020100 \\
\hline $\mathrm{H}$ & 0.78428900 & -1.04011000 & -0.87053100 \\
\hline $\mathrm{H}$ & 0.78428300 & -1.03912900 & 0.87167000 \\
\hline $\mathrm{C}$ & 1.95862400 & 0.54578900 & -0.00033100 \\
\hline $\mathrm{H}$ & 1.90560100 & 1.19487100 & 0.87107100 \\
\hline $\mathrm{H}$ & 1.90552200 & 1.19381400 & -0.87255100 \\
\hline $\mathrm{C}$ & 3.26807500 & -0.18751700 & -0.00002800 \\
\hline $\mathrm{H}$ & 3.68510600 & -0.56218400 & -0.91844200 \\
\hline $\mathrm{H}$ & 3.68966700 & -0.55396200 & 0.91958400 \\
\hline \multicolumn{4}{|l|}{$\mathrm{TS}_{18}$} \\
\hline \multicolumn{4}{|c|}{ Cartesian Coordinates $(\AA)$} \\
\hline $\mathrm{C}$ & -3.79505900 & -0.82060200 & 0.14680000 \\
\hline $\mathrm{H}$ & -3.48376700 & -1.83867200 & -0.06999500 \\
\hline
\end{tabular}




\begin{tabular}{crrr}
\hline $\mathrm{H}$ & -3.93447500 & -0.74052100 & 1.22153300 \\
$\mathrm{C}$ & -2.76289900 & 0.18970300 & -0.35278500 \\
$\mathrm{H}$ & -3.11861900 & 1.19847500 & -0.15110400 \\
$\mathrm{H}$ & -2.67310800 & 0.10784000 & -1.43421800 \\
$\mathrm{C}$ & -1.38608300 & -0.00168200 & 0.28511200 \\
$\mathrm{H}$ & -1.03065100 & -1.00896100 & 0.08276900 \\
$\mathrm{H}$ & -1.47701800 & 0.07951300 & 1.36716900 \\
$\mathrm{C}$ & -0.35256300 & 1.01192500 & -0.21032800 \\
$\mathrm{H}$ & -0.70581900 & 2.02252000 & 0.00350300 \\
$\mathrm{H}$ & -0.26157700 & 0.94796700 & -1.29282000 \\
$\mathrm{C}$ & 1.01857800 & 0.84691600 & 0.41558400 \\
$\mathrm{H}$ & 1.43297600 & -0.35907000 & 0.04039400 \\
$\mathrm{H}$ & 0.99120600 & 0.71914100 & 1.49155100 \\
$\mathrm{C}$ & 2.09320700 & 1.80756900 & -0.04524300 \\
$\mathrm{H}$ & 1.84745200 & 2.82457800 & 0.25662000 \\
$\mathrm{H}$ & 3.05660000 & 1.56050700 & 0.38706500 \\
$\mathrm{H}$ & 2.18944800 & 1.80205300 & -1.12694100 \\
$\mathrm{O}$ & 1.82276700 & -1.43420900 & -0.32943900 \\
$\mathrm{O}$ & 3.10111000 & -1.51184200 & 0.16604400 \\
$\mathrm{H}$ & 3.64578700 & -1.28777400 & -0.57887100 \\
$\mathrm{H}$ & -4.76054300 & -0.66215900 & -0.32433600 \\
\hline
\end{tabular}

\begin{tabular}{crrr}
\hline$s \mathrm{C}_{6} \mathrm{H}_{13}$ & \multicolumn{3}{c}{ Cartesian Coordinates $(\AA)$} \\
\hline $\mathrm{C}$ & 3.17266100 & 0.23537100 & -0.07411300 \\
$\mathrm{H}$ & 3.22852200 & 0.77436700 & -1.01607000 \\
$\mathrm{H}$ & 3.27581200 & 0.96189600 & 0.72748500 \\
$\mathrm{C}$ & 1.85757000 & -0.53441800 & 0.04433700 \\
$\mathrm{H}$ & 1.84820700 & -1.08977300 & 0.98029700 \\
$\mathrm{H}$ & 1.80136100 & -1.27563700 & -0.75059100 \\
$\mathrm{C}$ & 0.62740300 & 0.37172800 & -0.02014900 \\
$\mathrm{H}$ & 0.63607600 & 0.92773900 & -0.95568300 \\
$\mathrm{H}$ & 0.68509300 & 1.11238300 & 0.77612100 \\
$\mathrm{C}$ & -0.69380900 & -0.39210000 & 0.09751400 \\
$\mathrm{H}$ & -0.69496000 & -0.96046000 & 1.03273500 \\
$\mathrm{H}$ & -0.75575300 & -1.13658100 & -0.69564400 \\
$\mathrm{C}$ & -1.90643500 & 0.49258900 & 0.04197300 \\
$\mathrm{H}$ & -1.82844200 & 1.48118200 & 0.46664400 \\
$\mathrm{C}$ & -3.27552600 & -0.10157500 & -0.10753600 \\
$\mathrm{H}$ & -3.59212100 & -0.61812900 & 0.80127500 \\
$\mathrm{H}$ & -4.01894000 & 0.65949900 & -0.32147300 \\
$\mathrm{H}$ & -3.30283800 & -0.83241000 & -0.91198400 \\
$\mathrm{H}$ & 4.02680400 & -0.43364600 & -0.02526800 \\
\hline
\end{tabular}

$i \mathrm{C}_{6} \mathrm{H}_{14}$

Cartesian Coordinates $(\AA)$ 


\begin{tabular}{|c|c|c|c|}
\hline $\mathrm{C}$ & 2.83646200 & -0.21739000 & 0.14316600 \\
\hline $\mathrm{H}$ & 2.89738600 & -0.29810100 & 1.22529100 \\
\hline $\mathrm{H}$ & 3.66418200 & 0.40272400 & -0.18845100 \\
\hline $\mathrm{H}$ & 2.98224800 & -1.21199700 & -0.26964400 \\
\hline $\mathrm{C}$ & 1.49396600 & 0.37142800 & -0.29148900 \\
\hline $\mathrm{H}$ & 1.40852100 & 1.38054200 & 0.10118400 \\
\hline $\mathrm{H}$ & 1.47524600 & 0.46090700 & -1.37633900 \\
\hline $\mathrm{C}$ & 0.30556900 & -0.47757400 & 0.16756400 \\
\hline $\mathrm{H}$ & 0.29094700 & -0.51567500 & 1.25703600 \\
\hline $\mathrm{H}$ & 0.46586200 & -1.50123100 & -0.16735200 \\
\hline $\mathrm{C}$ & -1.06998000 & -0.01358500 & -0.33562500 \\
\hline $\mathrm{H}$ & -1.02485900 & 0.04341100 & -1.42316100 \\
\hline $\mathrm{C}$ & -2.14580400 & -1.04014100 & 0.03297800 \\
\hline $\mathrm{H}$ & -2.23450700 & -1.14071200 & 1.11237000 \\
\hline $\mathrm{H}$ & -3.11855900 & -0.74612300 & -0.35120200 \\
\hline $\mathrm{H}$ & -1.91244800 & -2.02086900 & -0.37184600 \\
\hline $\mathrm{C}$ & -1.45542500 & 1.37208400 & 0.19313500 \\
\hline $\mathrm{H}$ & -1.47497100 & 1.38013000 & 1.28091700 \\
\hline $\mathrm{H}$ & -0.76343000 & 2.14146800 & -0.13188200 \\
\hline $\mathrm{H}$ & -2.44433900 & 1.65658800 & -0.15529700 \\
\hline \multicolumn{4}{|l|}{$\mathrm{TS}_{19}$} \\
\hline \multicolumn{4}{|c|}{ Cartesian Coordinates $(\AA)$} \\
\hline $\mathrm{C}$ & -3.11036100 & 0.60452000 & 0.21578500 \\
\hline $\mathrm{H}$ & -2.91862200 & 1.40144400 & 0.92932600 \\
\hline $\mathrm{H}$ & -3.61158700 & -0.19929300 & 0.74822800 \\
\hline $\mathrm{C}$ & -1.81470500 & 0.11508900 & -0.43165700 \\
\hline $\mathrm{H}$ & -1.35000700 & 0.93292400 & -0.97740700 \\
\hline $\mathrm{H}$ & -2.05422900 & -0.64635500 & -1.16785900 \\
\hline $\mathrm{C}$ & -0.82909000 & -0.44247000 & 0.59912200 \\
\hline $\mathrm{H}$ & -1.27328100 & -1.31913300 & 1.07609700 \\
\hline $\mathrm{H}$ & -0.69156400 & 0.28833900 & 1.39254300 \\
\hline $\mathrm{C}$ & 0.54417300 & -0.84242600 & 0.07395100 \\
\hline $\mathrm{H}$ & 1.07513000 & 0.27284300 & -0.39495400 \\
\hline $\mathrm{C}$ & 1.50972100 & -1.24943600 & 1.17249200 \\
\hline $\mathrm{H}$ & 1.18654900 & -2.18552400 & 1.62769300 \\
\hline $\mathrm{H}$ & 2.51031200 & -1.39849000 & 0.78176000 \\
\hline $\mathrm{H}$ & 1.56070100 & -0.49846100 & 1.95280900 \\
\hline $\mathrm{C}$ & 0.55953900 & -1.77267200 & -1.12656700 \\
\hline $\mathrm{H}$ & 0.09567800 & -2.72504900 & -0.86957900 \\
\hline $\mathrm{H}$ & 0.02327900 & -1.36073400 & -1.97298500 \\
\hline $\mathrm{H}$ & 1.57659600 & -1.97613100 & -1.44377100 \\
\hline $\mathrm{O}$ & 1.61688700 & 1.27721900 & -0.80508700 \\
\hline $\mathrm{O}$ & 1.59039000 & 2.12863700 & 0.27269300 \\
\hline $\mathrm{H}$ & 0.85779900 & 2.70404000 & 0.08830400 \\
\hline
\end{tabular}




\begin{tabular}{crrr}
\hline $\mathrm{H}$ & -3.80063500 & 0.98710500 & -0.52981100 \\
\hline$t \mathrm{C}_{6} \mathrm{H}_{13}$ & & & \\
& \multicolumn{3}{c}{ Cartesian Coordinates $(\AA)$} \\
\hline $\mathrm{C}$ & 2.82508000 & -0.21915800 & 0.05856800 \\
$\mathrm{H}$ & 2.94630800 & -1.20121500 & -0.39060000 \\
$\mathrm{H}$ & 2.95298600 & -0.33004500 & 1.13218200 \\
$\mathrm{C}$ & 1.45855600 & 0.37837900 & -0.27651600 \\
$\mathrm{H}$ & 1.36820400 & 0.49598600 & -1.35409800 \\
$\mathrm{H}$ & 1.39560300 & 1.37556400 & 0.14944800 \\
$\mathrm{C}$ & 0.30243200 & -0.48715700 & 0.23677300 \\
$\mathrm{H}$ & 0.37079300 & -0.54850800 & 1.32874500 \\
$\mathrm{H}$ & 0.44444700 & -1.50450800 & -0.12179200 \\
$\mathrm{C}$ & -1.08364400 & -0.02844100 & -0.14890500 \\
$\mathrm{C}$ & -2.18082300 & -1.05681300 & -0.09263500 \\
$\mathrm{H}$ & -2.48328600 & -1.26048100 & 0.93846800 \\
$\mathrm{H}$ & -3.06722400 & -0.72336000 & -0.62410400 \\
$\mathrm{H}$ & -1.86588100 & -2.00182300 & -0.52496600 \\
$\mathrm{C}$ & -1.47710100 & 1.40350500 & 0.09878000 \\
$\mathrm{H}$ & -1.55547800 & 1.61283600 & 1.16940500 \\
$\mathrm{H}$ & -0.75939000 & 2.10580600 & -0.31102400 \\
$\mathrm{H}$ & -2.44393800 & 1.62547800 & -0.34205000 \\
$\mathrm{H}$ & 3.62985800 & 0.41237500 & -0.30600500 \\
\hline
\end{tabular}

$n \mathrm{C}_{7} \mathrm{H}_{16}$

Cartesian Coordinates $(\AA)$

\begin{tabular}{lrrr}
\hline $\mathrm{C}$ & -3.82877300 & -0.35308000 & -0.00000300 \\
$\mathrm{H}$ & -3.87381300 & -0.99321900 & -0.87703900 \\
$\mathrm{H}$ & -4.71853600 & 0.26984900 & -0.00000700 \\
$\mathrm{H}$ & -3.87382300 & -0.99322200 & 0.87703100 \\
$\mathrm{C}$ & -2.55703500 & 0.49449000 & 0.00000500 \\
$\mathrm{H}$ & -2.55967000 & 1.14762400 & -0.87050400 \\
$\mathrm{H}$ & -2.55967100 & 1.14761200 & 0.87052200 \\
$\mathrm{C}$ & -1.27808200 & -0.34451600 & 0.00000000 \\
$\mathrm{H}$ & -1.27912500 & -0.99873200 & -0.87084300 \\
$\mathrm{H}$ & -1.27912200 & -0.99873300 & 0.87084300 \\
$\mathrm{C}$ & 0.00000000 & 0.49585000 & -0.00000200 \\
$\mathrm{H}$ & 0.00000000 & 1.14990900 & 0.87087500 \\
$\mathrm{H}$ & 0.00000000 & 1.14990300 & -0.87088300 \\
$\mathrm{C}$ & 1.27808200 & -0.34451600 & 0.00000200 \\
$\mathrm{H}$ & 1.27912300 & -0.99873700 & -0.87083800 \\
$\mathrm{H}$ & 1.27912400 & -0.99872800 & 0.87084800 \\
$\mathrm{C}$ & 2.55703500 & 0.49449000 & -0.00000300 \\
$\mathrm{H}$ & 2.55967100 & 1.14762200 & 0.87050700 \\
$\mathrm{H}$ & 2.55967000 & 1.14761300 & -0.87051900 \\
\hline
\end{tabular}




\begin{tabular}{|c|c|c|c|}
\hline $\mathrm{C}$ & 3.82877300 & -0.35308000 & 0.00000100 \\
\hline $\mathrm{H}$ & 3.87381800 & -0.99322400 & -0.87703100 \\
\hline $\mathrm{H}$ & 4.71853600 & 0.26984900 & -0.00000200 \\
\hline $\mathrm{H}$ & 3.87381800 & -0.99321600 & 0.87703900 \\
\hline \multicolumn{4}{|l|}{$\mathrm{TS}_{20}$} \\
\hline \multicolumn{4}{|c|}{ Cartesian Coordinates $(\AA)$} \\
\hline $\mathrm{C}$ & -5.16802800 & -0.11252500 & 0.12222300 \\
\hline $\mathrm{H}$ & -5.26136100 & 0.17964700 & 1.16473600 \\
\hline $\mathrm{H}$ & -5.94876300 & -0.83730800 & -0.08867900 \\
\hline $\mathrm{H}$ & -5.36155600 & 0.76764700 & -0.48504300 \\
\hline $\mathrm{C}$ & -3.78288800 & -0.68985500 & -0.16764800 \\
\hline $\mathrm{H}$ & -3.63459800 & -1.58768400 & 0.42922400 \\
\hline $\mathrm{H}$ & -3.73403000 & -1.00398300 & -1.20847100 \\
\hline $\mathrm{C}$ & -2.65148600 & 0.29962400 & 0.11640200 \\
\hline $\mathrm{H}$ & -2.70316900 & 0.61366900 & 1.15793700 \\
\hline $\mathrm{H}$ & -2.80288900 & 1.19807400 & -0.48027300 \\
\hline $\mathrm{C}$ & -1.26181700 & -0.27093100 & -0.17159400 \\
\hline $\mathrm{H}$ & -1.20992900 & -0.58380900 & -1.21334200 \\
\hline $\mathrm{H}$ & -1.11098200 & -1.16926500 & 0.42508500 \\
\hline $\mathrm{C}$ & -0.13239400 & 0.72040300 & 0.11468000 \\
\hline $\mathrm{H}$ & -0.18063800 & 1.03381200 & 1.15580700 \\
\hline $\mathrm{H}$ & -0.27830500 & 1.61799100 & -0.48342100 \\
\hline $\mathrm{C}$ & 1.25930700 & 0.14020300 & -0.17447200 \\
\hline $\mathrm{H}$ & 1.30988200 & -0.16970300 & -1.21543300 \\
\hline $\mathrm{H}$ & 1.40835900 & -0.75122100 & 0.42930100 \\
\hline $\mathrm{C}$ & 2.36643100 & 1.13090100 & 0.11089800 \\
\hline $\mathrm{H}$ & 2.50353200 & 1.39198900 & 1.15145800 \\
\hline $\mathrm{H}$ & 3.53281400 & 0.53910800 & -0.17289700 \\
\hline $\mathrm{H}$ & 2.39505300 & 1.99747000 & -0.53667700 \\
\hline $\mathrm{O}$ & 4.55801300 & -0.01199500 & -0.40949100 \\
\hline $\mathrm{O}$ & 4.56885800 & -1.07983500 & 0.45371600 \\
\hline $\mathrm{H}$ & 4.28685300 & -1.80871100 & -0.08604700 \\
\hline \multicolumn{4}{|l|}{$p \mathrm{C}_{7} \mathrm{H}_{15}$} \\
\hline \multicolumn{4}{|c|}{ Cartesian Coordinates $(\AA)$} \\
\hline $\mathrm{C}$ & 3.75772400 & -0.34698200 & 0.00007400 \\
\hline $\mathrm{H}$ & 3.80590500 & -0.98661600 & 0.87733400 \\
\hline $\mathrm{H}$ & 4.64422100 & 0.28048300 & -0.00007800 \\
\hline $\mathrm{H}$ & 3.80590000 & -0.98703500 & -0.87688000 \\
\hline $\mathrm{C}$ & 2.48196800 & 0.49481300 & -0.00012100 \\
\hline $\mathrm{H}$ & 2.48124800 & 1.14800200 & 0.87038900 \\
\hline $\mathrm{H}$ & 2.48125300 & 1.14760000 & -0.87093300 \\
\hline $\mathrm{C}$ & 1.20712200 & -0.35061100 & 0.00007200 \\
\hline $\mathrm{H}$ & 1.21105800 & -1.00438300 & 0.87119600 \\
\hline $\mathrm{H}$ & 1.21102400 & -1.00471600 & -0.87080100 \\
\hline
\end{tabular}




\begin{tabular}{|c|c|c|c|}
\hline $\mathrm{C}$ & -0.07462700 & 0.48422500 & -0.00005600 \\
\hline $\mathrm{H}$ & -0.07788000 & 1.13780700 & -0.87129000 \\
\hline $\mathrm{H}$ & -0.07782000 & 1.13813500 & 0.87093500 \\
\hline $\mathrm{C}$ & -1.34772400 & -0.36348800 & 0.00014800 \\
\hline $\mathrm{H}$ & -1.35042700 & -1.01570600 & 0.87140700 \\
\hline $\mathrm{H}$ & -1.35040500 & -1.01622200 & -0.87073300 \\
\hline $\mathrm{C}$ & -2.63259800 & 0.48351500 & -0.00011900 \\
\hline $\mathrm{H}$ & -2.62494100 & 1.13398800 & -0.87211500 \\
\hline $\mathrm{H}$ & -2.62511300 & 1.13442100 & 0.87153900 \\
\hline $\mathrm{C}$ & -3.88759100 & -0.33953700 & -0.00004800 \\
\hline $\mathrm{H}$ & -4.28083400 & -0.73718100 & 0.91917400 \\
\hline $\mathrm{H}$ & -4.27882700 & -0.74018000 & -0.91884200 \\
\hline \multicolumn{4}{|l|}{$\mathrm{TS}_{21}$} \\
\hline \multicolumn{4}{|c|}{ Cartesian Coordinates $(\AA)$} \\
\hline $\mathrm{C}$ & -4.63842100 & -0.34801700 & -0.25587300 \\
\hline $\mathrm{H}$ & -4.97967200 & 0.66358100 & -0.05241400 \\
\hline $\mathrm{H}$ & -5.37282200 & -1.03338500 & 0.15681700 \\
\hline $\mathrm{H}$ & -4.63073700 & -0.48236400 & -1.33424600 \\
\hline $\mathrm{C}$ & -3.25332100 & -0.59474500 & 0.34120900 \\
\hline $\mathrm{H}$ & -2.95608900 & -1.62379600 & 0.15027600 \\
\hline $\mathrm{H}$ & -3.30285300 & -0.48726400 & 1.42303900 \\
\hline $\mathrm{C}$ & -2.18665300 & 0.35090300 & -0.21330500 \\
\hline $\mathrm{H}$ & -2.48607500 & 1.38070100 & -0.02181200 \\
\hline $\mathrm{H}$ & -2.14098800 & 0.24328300 & -1.29615200 \\
\hline $\mathrm{C}$ & -0.79752900 & 0.10666900 & 0.37799600 \\
\hline $\mathrm{H}$ & -0.49659200 & -0.91995900 & 0.18521600 \\
\hline $\mathrm{H}$ & -0.84247900 & 0.21488400 & 1.46047900 \\
\hline $\mathrm{C}$ & 0.26262200 & 1.05969700 & -0.17813300 \\
\hline $\mathrm{H}$ & -0.03469000 & 2.09014300 & 0.02571300 \\
\hline $\mathrm{H}$ & 0.30936400 & 0.96897700 & -1.26150600 \\
\hline $\mathrm{C}$ & 1.64736800 & 0.84289900 & 0.40021200 \\
\hline $\mathrm{H}$ & 1.99002200 & -0.38936200 & 0.03832600 \\
\hline $\mathrm{H}$ & 1.65507900 & 0.73994400 & 1.47917100 \\
\hline $\mathrm{C}$ & 2.74758600 & 1.74092000 & -0.12241600 \\
\hline $\mathrm{H}$ & 2.56168200 & 2.77474300 & 0.16477000 \\
\hline $\mathrm{H}$ & 3.71415200 & 1.45725000 & 0.27924100 \\
\hline $\mathrm{H}$ & 2.80189900 & 1.70779400 & -1.20655100 \\
\hline $\mathrm{O}$ & 2.31415200 & -1.48979300 & -0.32059400 \\
\hline $\mathrm{O}$ & 3.60996800 & -1.61314100 & 0.11679900 \\
\hline $\mathrm{H}$ & 4.12793400 & -1.43165200 & -0.65814600 \\
\hline \multicolumn{4}{|l|}{$s \mathrm{C}_{7} \mathrm{H}_{15}$} \\
\hline \multicolumn{4}{|c|}{ Cartesian Coordinates $(\AA)$} \\
\hline $\mathrm{C}$ & 3.79127500 & -0.32931100 & -0.00728900 \\
\hline $\mathrm{H}$ & 3.86088600 & -0.86602200 & 0.93513600 \\
\hline
\end{tabular}




\begin{tabular}{|c|c|c|c|}
\hline $\mathrm{H}$ & 4.67394800 & 0.29722400 & -0.09636700 \\
\hline $\mathrm{H}$ & 3.82543100 & -1.06378100 & -0.80755700 \\
\hline $\mathrm{C}$ & 2.51094800 & 0.50268500 & -0.07570800 \\
\hline $\mathrm{H}$ & 2.48877100 & 1.05320100 & -1.01420800 \\
\hline $\mathrm{H}$ & 2.52408600 & 1.24974200 & 0.71561600 \\
\hline $\mathrm{C}$ & 1.24131600 & -0.34167900 & 0.04615000 \\
\hline $\mathrm{H}$ & 1.26666200 & -0.89302100 & 0.98523600 \\
\hline $\mathrm{H}$ & 1.23168400 & -1.08985200 & -0.74543400 \\
\hline $\mathrm{C}$ & -0.04399900 & 0.48396600 & -0.02227400 \\
\hline $\mathrm{H}$ & -0.07233800 & 1.03472600 & -0.96039800 \\
\hline $\mathrm{H}$ & -0.03504400 & 1.23039800 & 0.77050300 \\
\hline $\mathrm{C}$ & -1.31329000 & -0.36304200 & 0.09966100 \\
\hline $\mathrm{H}$ & -1.27719400 & -0.92663600 & 1.03704500 \\
\hline $\mathrm{H}$ & -1.32777800 & -1.11302100 & -0.69055300 \\
\hline $\mathrm{C}$ & -2.58036900 & 0.44154700 & 0.04196300 \\
\hline $\mathrm{H}$ & -2.56674100 & 1.43342500 & 0.46596000 \\
\hline $\mathrm{C}$ & -3.90821700 & -0.23956000 & -0.10882200 \\
\hline $\mathrm{H}$ & -4.19220900 & -0.77474900 & 0.79998200 \\
\hline $\mathrm{H}$ & -4.69877400 & 0.47196300 & -0.32435000 \\
\hline $\mathrm{H}$ & -3.88737800 & -0.97123300 & -0.91270100 \\
\hline \multicolumn{4}{|l|}{$i \mathrm{C}_{7} \mathrm{H}_{16}$} \\
\hline \multicolumn{4}{|c|}{ Cartesian Coordinates $(\AA)$} \\
\hline $\mathrm{C}$ & 3.49317500 & 0.24499000 & -0.12320400 \\
\hline $\mathrm{H}$ & 3.56817500 & 0.37018900 & -1.20017800 \\
\hline $\mathrm{H}$ & 4.38170100 & -0.28312500 & 0.21034800 \\
\hline $\mathrm{H}$ & 3.50961000 & 1.23485900 & 0.32509800 \\
\hline $\mathrm{C}$ & 2.22160900 & -0.51297900 & 0.25727100 \\
\hline $\mathrm{H}$ & 2.25425200 & -1.51101800 & -0.17530300 \\
\hline $\mathrm{H}$ & 2.19308600 & -0.64956200 & 1.33670800 \\
\hline $\mathrm{C}$ & 0.94380400 & 0.19578400 & -0.19730100 \\
\hline $\mathrm{H}$ & 0.97046700 & 0.32046700 & -1.27941500 \\
\hline $\mathrm{H}$ & 0.92949600 & 1.19705500 & 0.22574100 \\
\hline $\mathrm{C}$ & -0.32767700 & -0.56160100 & 0.19425800 \\
\hline $\mathrm{H}$ & -0.38827700 & -0.62630400 & 1.28076500 \\
\hline $\mathrm{H}$ & -0.24217700 & -1.58610400 & -0.16432300 \\
\hline $\mathrm{C}$ & -1.63916500 & 0.03048700 & -0.34474800 \\
\hline $\mathrm{H}$ & -1.54810600 & 0.11032200 & -1.42794700 \\
\hline $\mathrm{C}$ & -2.81093600 & -0.90940900 & -0.04371300 \\
\hline $\mathrm{H}$ & -2.94896100 & -1.02865400 & 1.02857900 \\
\hline $\mathrm{H}$ & -3.74011400 & -0.52420100 & -0.45430000 \\
\hline $\mathrm{H}$ & -2.64575400 & -1.89619500 & -0.46697600 \\
\hline $\mathrm{C}$ & -1.92632100 & 1.43047200 & 0.20819300 \\
\hline $\mathrm{H}$ & -1.98616500 & 1.41336800 & 1.29441300 \\
\hline $\mathrm{H}$ & -1.16022100 & 2.14585300 & -0.07063300 \\
\hline
\end{tabular}




\begin{tabular}{|c|c|c|c|}
\hline $\mathrm{H}$ & -2.87394900 & 1.80658600 & -0.16711600 \\
\hline \multicolumn{4}{|l|}{$\mathrm{TS}_{22}$} \\
\hline \multicolumn{4}{|c|}{ Cartesian Coordinates $(\AA)$} \\
\hline $\mathrm{C}$ & 3.83563800 & 0.35723200 & 0.31005400 \\
\hline $\mathrm{H}$ & 3.63197300 & 1.27105200 & 0.86184200 \\
\hline $\mathrm{H}$ & 4.73613200 & 0.51842300 & -0.27498900 \\
\hline $\mathrm{H}$ & 4.04790400 & -0.42411100 & 1.03471000 \\
\hline $\mathrm{C}$ & 2.65582000 & -0.02382500 & -0.58353100 \\
\hline $\mathrm{H}$ & 2.49531500 & 0.75899100 & -1.32242100 \\
\hline $\mathrm{H}$ & 2.90362400 & -0.92366000 & -1.14322600 \\
\hline $\mathrm{C}$ & 1.36302700 & -0.25488200 & 0.20195500 \\
\hline $\mathrm{H}$ & 1.10730900 & 0.64996500 & 0.75007900 \\
\hline $\mathrm{H}$ & 1.53937300 & -1.02404200 & 0.94920000 \\
\hline $\mathrm{C}$ & 0.19393000 & -0.65901900 & -0.70050900 \\
\hline $\mathrm{H}$ & 0.42163200 & -1.61943200 & -1.16831600 \\
\hline $\mathrm{H}$ & 0.10821100 & 0.05486200 & -1.51617800 \\
\hline $\mathrm{C}$ & -1.16784000 & -0.78022200 & -0.02749400 \\
\hline $\mathrm{H}$ & -1.43969400 & 0.43010400 & 0.42683100 \\
\hline $\mathrm{C}$ & -2.29767300 & -1.04351100 & -1.00674700 \\
\hline $\mathrm{H}$ & -2.19537300 & -2.03916200 & -1.43818400 \\
\hline $\mathrm{H}$ & -3.26363600 & -0.99192700 & -0.51647600 \\
\hline $\mathrm{H}$ & -2.29280700 & -0.32526000 & -1.81889300 \\
\hline $\mathrm{C}$ & -1.22693600 & -1.64673700 & 1.21848700 \\
\hline $\mathrm{H}$ & -0.96981100 & -2.67693800 & 0.97184800 \\
\hline $\mathrm{H}$ & -0.54200900 & -1.30915400 & 1.98698400 \\
\hline $\mathrm{H}$ & -2.22623400 & -1.64811200 & 1.64020200 \\
\hline $\mathrm{O}$ & -1.74792300 & 1.53182800 & 0.82921100 \\
\hline $\mathrm{O}$ & -1.67271200 & 2.32392100 & -0.29069100 \\
\hline $\mathrm{H}$ & -0.83261200 & 2.75819300 & -0.20446000 \\
\hline
\end{tabular}

${ }_{t} \mathrm{C}_{7} \mathrm{H}_{15}$

Cartesian Coordinates $(\AA)$

\begin{tabular}{rrrr}
\hline $\mathrm{C}$ & 3.45555700 & 0.25431000 & -0.18577000 \\
$\mathrm{H}$ & 3.48163300 & 0.42924700 & -1.25802400 \\
$\mathrm{H}$ & 4.35888400 & -0.28559100 & 0.08273300 \\
$\mathrm{H}$ & 3.49078000 & 1.22245600 & 0.30669100 \\
$\mathrm{C}$ & 2.20361300 & -0.52486400 & 0.21613300 \\
$\mathrm{H}$ & 2.21825200 & -1.50172000 & -0.26323800 \\
$\mathrm{H}$ & 2.22392900 & -0.71110600 & 1.28834500 \\
$\mathrm{C}$ & 0.90663200 & 0.20011800 & -0.14832600 \\
$\mathrm{H}$ & 0.87936700 & 0.37214600 & -1.22293900 \\
$\mathrm{H}$ & 0.90926000 & 1.18084900 & 0.32060400 \\
$\mathrm{C}$ & -0.34436700 & -0.57952400 & 0.27126900 \\
$\mathrm{H}$ & -0.33996000 & -0.68766400 & 1.36162500 \\
\hline
\end{tabular}




\begin{tabular}{lrrr}
\hline $\mathrm{H}$ & -0.27312400 & -1.59120000 & -0.12243100 \\
$\mathrm{C}$ & -1.66372100 & 0.01398100 & -0.16216700 \\
$\mathrm{C}$ & -2.84810800 & -0.91374000 & -0.19547900 \\
$\mathrm{H}$ & -3.21839500 & -1.12323300 & 0.81206700 \\
$\mathrm{H}$ & -3.67429700 & -0.48543000 & -0.75527200 \\
$\mathrm{H}$ & -2.59582300 & -1.86847200 & -0.64716800 \\
$\mathrm{C}$ & -1.94228500 & 1.46640500 & 0.11965300 \\
$\mathrm{H}$ & -2.05456300 & 1.64697100 & 1.19250900 \\
$\mathrm{H}$ & -1.14663600 & 2.11489100 & -0.23061700 \\
$\mathrm{H}$ & -2.86323900 & 1.78774100 & -0.35677100 \\
\hline
\end{tabular}

$n \mathrm{C}_{8} \mathrm{H}_{18}$

Cartesian Coordinates $(\AA)$

\begin{tabular}{|c|c|c|c|}
\hline \\
\hline $\mathrm{C}$ & -4.48121600 & 0.25660000 & 0.00000400 \\
\hline $\mathrm{H}$ & -4.55085000 & 0.89454300 & -0.87702700 \\
\hline $\mathrm{H}$ & -4.55084000 & 0.89453600 & 0.87704200 \\
\hline $\mathrm{H}$ & -5.34639600 & -0.40006700 & 0.00000600 \\
\hline $\mathrm{C}$ & -3.17781000 & -0.54138200 & -0.00000500 \\
\hline $\mathrm{H}$ & -3.15534800 & -1.19413900 & 0.87050200 \\
\hline $\mathrm{H}$ & -3.15535000 & -1.19412500 & -0.87052300 \\
\hline $\mathrm{C}$ & -1.93200400 & 0.34612800 & 0.00000000 \\
\hline $\mathrm{H}$ & -1.95822400 & 0.99982000 & 0.87085200 \\
\hline $\mathrm{H}$ & -1.95822100 & 0.99982300 & -0.87085000 \\
\hline $\mathrm{C}$ & -0.62251600 & -0.44443100 & 0.00000100 \\
\hline $\mathrm{H}$ & -0.59743800 & -1.09800800 & -0.87084100 \\
\hline $\mathrm{H}$ & -0.59743700 & -1.09800200 & 0.87084700 \\
\hline $\mathrm{C}$ & 0.62251600 & 0.44443100 & -0.00000300 \\
\hline $\mathrm{H}$ & 0.59743700 & 1.09800900 & 0.87083900 \\
\hline $\mathrm{H}$ & 0.59743800 & 1.09800100 & -0.87085000 \\
\hline $\mathrm{C}$ & 1.93200400 & -0.34612800 & 0.00000200 \\
\hline $\mathrm{H}$ & 1.95822300 & -0.99982500 & -0.87084600 \\
\hline $\mathrm{H}$ & 1.95822200 & -0.99981700 & 0.87085600 \\
\hline $\mathrm{C}$ & 3.17781000 & 0.54138200 & -0.00000200 \\
\hline $\mathrm{H}$ & 3.15534900 & 1.19413600 & 0.87050800 \\
\hline $\mathrm{H}$ & 3.15534900 & 1.19412800 & -0.87051800 \\
\hline $\mathrm{C}$ & 4.48121600 & -0.25660000 & 0.00000200 \\
\hline $\mathrm{H}$ & 4.55084500 & -0.89454300 & -0.87703000 \\
\hline $\mathrm{H}$ & 5.34639600 & 0.40006700 & -0.00000100 \\
\hline $\mathrm{H}$ & 4.55084500 & -0.89453600 & 0.87703900 \\
\hline \multicolumn{4}{|l|}{$\mathrm{TS}_{23}$} \\
\hline \multicolumn{4}{|c|}{ Cartesian Coordinates $(\AA)$} \\
\hline $\mathrm{C}$ & -5.73254600 & -0.69094700 & -0.08455200 \\
\hline $\mathrm{H}$ & -5.66546300 & -1.60977300 & 0.49184400 \\
\hline $\mathrm{H}$ & -5.76625100 & -0.96373900 & -1.13593900 \\
\hline $\mathrm{H}$ & -6.67627400 & -0.21392300 & 0.16319500 \\
\hline
\end{tabular}




\begin{tabular}{|c|c|c|c|}
\hline $\mathrm{C}$ & -4.55040400 & 0.23242400 & 0.20870600 \\
\hline $\mathrm{H}$ & -4.66563500 & 1.15560800 & -0.35602400 \\
\hline $\mathrm{H}$ & -4.56550200 & 0.51420800 & 1.25983000 \\
\hline $\mathrm{C}$ & -3.19906200 & -0.40101800 & -0.12649800 \\
\hline $\mathrm{H}$ & -3.18699900 & -0.68368100 & -1.17825800 \\
\hline $\mathrm{H}$ & -3.08693000 & -1.32568400 & 0.43800200 \\
\hline $\mathrm{C}$ & -2.01032700 & 0.51654100 & 0.16441800 \\
\hline $\mathrm{H}$ & -2.02289800 & 0.79818000 & 1.21639200 \\
\hline $\mathrm{H}$ & -2.12350100 & 1.44122100 & -0.39974400 \\
\hline $\mathrm{C}$ & -0.66007600 & -0.11870000 & -0.17227000 \\
\hline $\mathrm{H}$ & -0.64591000 & -0.39919400 & -1.22417700 \\
\hline $\mathrm{H}$ & -0.54623200 & -1.04324800 & 0.39134500 \\
\hline $\mathrm{C}$ & 0.52779100 & 0.79980300 & 0.12062500 \\
\hline $\mathrm{H}$ & 0.51522200 & 1.08347500 & 1.17164200 \\
\hline $\mathrm{H}$ & 0.41993100 & 1.72229200 & -0.44735900 \\
\hline $\mathrm{C}$ & 1.87851400 & 0.15169800 & -0.21390200 \\
\hline $\mathrm{H}$ & 1.89351100 & -0.13414200 & -1.26200600 \\
\hline $\mathrm{H}$ & 1.99629600 & -0.76228600 & 0.36087500 \\
\hline $\mathrm{C}$ & 3.04389400 & 1.07403400 & 0.06890900 \\
\hline $\mathrm{H}$ & 3.20680400 & 1.31535200 & 1.11135500 \\
\hline $\mathrm{H}$ & 4.15462900 & 0.40007000 & -0.24946200 \\
\hline $\mathrm{H}$ & 3.11770200 & 1.94616500 & -0.56765300 \\
\hline $\mathrm{O}$ & 5.11587800 & -0.24347100 & -0.52199700 \\
\hline $\mathrm{O}$ & 5.14397400 & -1.22173000 & 0.44142000 \\
\hline $\mathrm{H}$ & 5.78198500 & -0.90230700 & 1.06814700 \\
\hline \multicolumn{4}{|l|}{$p \mathrm{C}_{8} \mathrm{H}_{17}$} \\
\hline \multicolumn{4}{|c|}{ Cartesian Coordinates $(\AA)$} \\
\hline $\mathrm{C}$ & -4.40628700 & 0.26122700 & -0.00004800 \\
\hline $\mathrm{H}$ & -4.47423300 & 0.89890100 & -0.87743600 \\
\hline $\mathrm{H}$ & -4.47419300 & 0.89968000 & 0.87677600 \\
\hline $\mathrm{H}$ & -5.27300900 & -0.39330900 & 0.00026300 \\
\hline $\mathrm{C}$ & -3.10512600 & -0.54074100 & 0.00027900 \\
\hline $\mathrm{H}$ & -3.08425800 & -1.19304300 & 0.87121600 \\
\hline $\mathrm{H}$ & -3.08430100 & -1.19381800 & -0.87007600 \\
\hline $\mathrm{C}$ & -1.85695400 & 0.34349500 & -0.00015200 \\
\hline $\mathrm{H}$ & -1.88116300 & 0.99750200 & 0.87054600 \\
\hline $\mathrm{H}$ & -1.88114700 & 0.99664500 & -0.87149100 \\
\hline $\mathrm{C}$ & -0.54999500 & -0.45127800 & 0.00025400 \\
\hline $\mathrm{H}$ & -0.52679000 & -1.10515300 & -0.87036700 \\
\hline $\mathrm{H}$ & -0.52672100 & -1.10410300 & 0.87165300 \\
\hline $\mathrm{C}$ & 0.69739300 & 0.43436700 & -0.00033500 \\
\hline $\mathrm{H}$ & 0.67453600 & 1.08824200 & 0.87035500 \\
\hline $\mathrm{H}$ & 0.67440600 & 1.08720000 & -0.87180800 \\
\hline $\mathrm{C}$ & 2.00342800 & -0.36163700 & 0.00006900 \\
\hline
\end{tabular}




\begin{tabular}{|c|c|c|c|}
\hline $\mathrm{H}$ & 2.03236100 & -1.01373900 & -0.87081300 \\
\hline $\mathrm{H}$ & 2.03229500 & -1.01322200 & 0.87134400 \\
\hline $\mathrm{C}$ & 3.25332100 & 0.53619200 & -0.00015200 \\
\hline $\mathrm{H}$ & 3.21971100 & 1.18634900 & 0.87142700 \\
\hline $\mathrm{H}$ & 3.21970900 & 1.18577400 & -0.87219500 \\
\hline $\mathrm{C}$ & 4.54029200 & -0.23591800 & 0.00001500 \\
\hline $\mathrm{H}$ & 4.94591500 & -0.62273500 & -0.91848700 \\
\hline $\mathrm{H}$ & 4.95044400 & -0.61541500 & 0.91952100 \\
\hline \multicolumn{4}{|l|}{$\mathrm{TS}_{24}$} \\
\hline \multicolumn{4}{|c|}{ Cartesian Coordinates $(\AA)$} \\
\hline $\mathrm{C}$ & 5.22212200 & 0.74591300 & 0.15112600 \\
\hline $\mathrm{H}$ & 5.04945400 & 1.79474800 & -0.07466300 \\
\hline $\mathrm{H}$ & 5.32962200 & 0.65463700 & 1.22868200 \\
\hline $\mathrm{H}$ & 6.16834800 & 0.46227200 & -0.30032200 \\
\hline $\mathrm{C}$ & 4.07769600 & -0.12559300 & -0.36544400 \\
\hline $\mathrm{H}$ & 4.01944300 & -0.03855800 & -1.44871400 \\
\hline $\mathrm{H}$ & 4.29732000 & -1.17061600 & -0.15489500 \\
\hline $\mathrm{C}$ & 2.72415500 & 0.24101000 & 0.24543900 \\
\hline $\mathrm{H}$ & 2.78535000 & 0.15532100 & 1.32952400 \\
\hline $\mathrm{H}$ & 2.50700500 & 1.28706700 & 0.03490700 \\
\hline $\mathrm{C}$ & 1.57342300 & -0.62678100 & -0.26650700 \\
\hline $\mathrm{H}$ & 1.79011400 & -1.67303000 & -0.05439400 \\
\hline $\mathrm{H}$ & 1.51413200 & -0.54142400 & -1.35057300 \\
\hline $\mathrm{C}$ & 0.22100800 & -0.25476200 & 0.34341500 \\
\hline $\mathrm{H}$ & 0.00127500 & 0.78819600 & 0.12986100 \\
\hline $\mathrm{H}$ & 0.27863800 & -0.34020200 & 1.42731300 \\
\hline $\mathrm{C}$ & -0.92454900 & -1.13039900 & -0.16883700 \\
\hline $\mathrm{H}$ & -0.70868700 & -2.17659900 & 0.05623300 \\
\hline $\mathrm{H}$ & -0.98531800 & -1.06249600 & -1.25319500 \\
\hline $\mathrm{C}$ & -2.27508700 & -0.78627000 & 0.42834100 \\
\hline $\mathrm{H}$ & -2.52265900 & 0.46097700 & 0.04111900 \\
\hline $\mathrm{H}$ & -2.25285000 & -0.65700400 & 1.50426600 \\
\hline $\mathrm{C}$ & -3.45541500 & -1.60292100 & -0.05123400 \\
\hline $\mathrm{H}$ & -3.34930800 & -2.64122700 & 0.25939600 \\
\hline $\mathrm{H}$ & -4.38731900 & -1.23069100 & 0.35991600 \\
\hline $\mathrm{H}$ & -3.52822400 & -1.59197800 & -1.13474000 \\
\hline $\mathrm{O}$ & -2.76266200 & 1.57537800 & -0.34019300 \\
\hline $\mathrm{O}$ & -4.03815800 & 1.81264600 & 0.10976800 \\
\hline $\mathrm{H}$ & -4.57988700 & 1.65523900 & -0.65411500 \\
\hline \multicolumn{4}{|l|}{$s \mathrm{C}_{8} \mathrm{H}_{17}$} \\
\hline \multicolumn{4}{|c|}{ Cartesian Coordinates $(\AA)$} \\
\hline $\mathrm{C}$ & -4.43263100 & 0.27609100 & 0.06452000 \\
\hline $\mathrm{H}$ & -4.48379800 & 0.83939500 & 0.99248100 \\
\hline $\mathrm{H}$ & -4.51104700 & 0.98499600 & -0.75545600 \\
\hline
\end{tabular}




\begin{tabular}{|c|c|c|c|}
\hline $\mathrm{H}$ & -5.30245000 & -0.37308000 & 0.02400100 \\
\hline $\mathrm{C}$ & -3.13552200 & -0.52770600 & -0.02265600 \\
\hline $\mathrm{H}$ & -3.10439800 & -1.25060700 & 0.79030700 \\
\hline $\mathrm{H}$ & -3.13133900 & -1.10580000 & -0.94476500 \\
\hline $\mathrm{C}$ & -1.88322400 & 0.34902100 & 0.03111100 \\
\hline $\mathrm{H}$ & -1.91822300 & 1.07353200 & -0.78147800 \\
\hline $\mathrm{H}$ & -1.89017700 & 0.92715700 & 0.95416100 \\
\hline $\mathrm{C}$ & -0.58037500 & -0.44746300 & -0.05718500 \\
\hline $\mathrm{H}$ & -0.57426900 & -1.02511100 & -0.98052600 \\
\hline $\mathrm{H}$ & -0.54671600 & -1.17207500 & 0.75524600 \\
\hline $\mathrm{C}$ & 0.67015700 & 0.43119200 & -0.00216600 \\
\hline $\mathrm{H}$ & 0.66803100 & 1.00867500 & 0.92015500 \\
\hline $\mathrm{H}$ & 0.63831100 & 1.15419200 & -0.81576600 \\
\hline $\mathrm{C}$ & 1.97350100 & -0.36688400 & -0.09018900 \\
\hline $\mathrm{H}$ & 1.96849400 & -0.95751300 & -1.01148800 \\
\hline $\mathrm{H}$ & 2.01111300 & -1.09324700 & 0.72105400 \\
\hline $\mathrm{C}$ & 3.20662900 & 0.48960800 & -0.04543800 \\
\hline $\mathrm{H}$ & 3.15716600 & 1.46749200 & -0.49829900 \\
\hline $\mathrm{C}$ & 4.55932800 & -0.13248800 & 0.13630800 \\
\hline $\mathrm{H}$ & 4.87340200 & -0.68159500 & -0.75411400 \\
\hline $\mathrm{H}$ & 5.31848300 & 0.61639800 & 0.33774100 \\
\hline $\mathrm{H}$ & 4.56024100 & -0.84102500 & 0.96091700 \\
\hline \multicolumn{4}{|l|}{$i \mathrm{C}_{8} \mathrm{H}_{18}$} \\
\hline \multicolumn{4}{|c|}{ Cartesian Coordinates $(\AA)$} \\
\hline $\mathrm{C}$ & 4.19311400 & -0.22002400 & 0.15725600 \\
\hline $\mathrm{H}$ & 4.24652000 & -0.37237400 & 1.23197500 \\
\hline $\mathrm{H}$ & 5.04677900 & 0.38540300 & -0.13318600 \\
\hline $\mathrm{H}$ & 4.29986200 & -1.19130900 & -0.31842500 \\
\hline $\mathrm{C}$ & 2.87742800 & 0.44827300 & -0.24094700 \\
\hline $\mathrm{H}$ & 2.87035300 & 0.61739000 & -1.31609200 \\
\hline $\mathrm{H}$ & 2.81813400 & 1.43120100 & 0.22253400 \\
\hline $\mathrm{C}$ & 1.64703500 & -0.37176800 & 0.15073800 \\
\hline $\mathrm{H}$ & 1.65563800 & -0.53827100 & 1.22721800 \\
\hline $\mathrm{H}$ & 1.71207000 & -1.35677700 & -0.30942600 \\
\hline $\mathrm{C}$ & 0.32541700 & 0.28814200 & -0.24850800 \\
\hline $\mathrm{H}$ & 0.31290500 & 0.44073600 & -1.32718400 \\
\hline $\mathrm{H}$ & 0.27641900 & 1.27652500 & 0.20113300 \\
\hline $\mathrm{C}$ & -0.89740400 & -0.53791600 & 0.15950700 \\
\hline $\mathrm{H}$ & -0.92258400 & -0.63201300 & 1.24523600 \\
\hline $\mathrm{H}$ & -0.77525500 & -1.54814300 & -0.22770300 \\
\hline $\mathrm{C}$ & -2.25041200 & 0.00473500 & -0.32625100 \\
\hline $\mathrm{H}$ & -2.19570200 & 0.11446800 & -1.40929700 \\
\hline $\mathrm{C}$ & -3.36790800 & -0.99605400 & -0.01525700 \\
\hline $\mathrm{H}$ & -3.46864300 & -1.14648900 & 1.05726100 \\
\hline
\end{tabular}




\begin{tabular}{|c|c|c|c|}
\hline $\mathrm{H}$ & -4.32567800 & -0.64572200 & -0.38982300 \\
\hline $\mathrm{H}$ & -3.16957000 & -1.96389000 & -0.46706900 \\
\hline $\mathrm{C}$ & -2.58524500 & 1.37617000 & 0.26977000 \\
\hline $\mathrm{H}$ & -2.61081100 & 1.33076400 & 1.35652500 \\
\hline $\mathrm{H}$ & -1.86217800 & 2.13329200 & -0.01370200 \\
\hline $\mathrm{H}$ & -3.56041700 & 1.71587500 & -0.06781700 \\
\hline \multicolumn{4}{|l|}{$\mathrm{TS}_{25}$} \\
\hline \multicolumn{4}{|c|}{ Cartesian Coordinates $(\AA)$} \\
\hline $\mathrm{C}$ & -4.81369800 & 0.37501700 & -0.21018600 \\
\hline $\mathrm{H}$ & -5.13524500 & -0.66307700 & -0.21943700 \\
\hline $\mathrm{H}$ & -4.81870800 & 0.73050800 & -1.23704100 \\
\hline $\mathrm{H}$ & -5.55585700 & 0.94563800 & 0.34029800 \\
\hline $\mathrm{C}$ & -3.42726700 & 0.51843200 & 0.41684600 \\
\hline $\mathrm{H}$ & -3.14981200 & 1.57034100 & 0.44141200 \\
\hline $\mathrm{H}$ & -3.46483400 & 0.18816200 & 1.45325600 \\
\hline $\mathrm{C}$ & -2.35001100 & -0.27141800 & -0.32816900 \\
\hline $\mathrm{H}$ & -2.31889600 & 0.05635900 & -1.36602200 \\
\hline $\mathrm{H}$ & -2.62815400 & -1.32452600 & -0.34919900 \\
\hline $\mathrm{C}$ & -0.95821700 & -0.12559300 & 0.29051900 \\
\hline $\mathrm{H}$ & -1.00188700 & -0.43612200 & 1.33138200 \\
\hline $\mathrm{H}$ & -0.67256700 & 0.92374300 & 0.29083500 \\
\hline $\mathrm{C}$ & 0.09988200 & -0.94300000 & -0.45601200 \\
\hline $\mathrm{H}$ & 0.05355500 & -0.69928200 & -1.51450300 \\
\hline $\mathrm{H}$ & -0.14778100 & -2.00420800 & -0.38079600 \\
\hline $\mathrm{C}$ & 1.54030100 & -0.76574700 & 0.01061400 \\
\hline $\mathrm{H}$ & 1.78670000 & 0.51462200 & -0.20266500 \\
\hline $\mathrm{C}$ & 2.55129700 & -1.46751900 & -0.87814300 \\
\hline $\mathrm{H}$ & 2.39879400 & -1.21925800 & -1.92276800 \\
\hline $\mathrm{H}$ & 3.56643400 & -1.19584100 & -0.61150500 \\
\hline $\mathrm{H}$ & 2.45519700 & -2.54821400 & -0.77393800 \\
\hline $\mathrm{C}$ & 1.79434200 & -0.96647900 & 1.49412300 \\
\hline $\mathrm{H}$ & 1.19539800 & -0.30365100 & 2.10758600 \\
\hline $\mathrm{H}$ & 1.55339200 & -1.99015800 & 1.78081200 \\
\hline $\mathrm{H}$ & 2.83875800 & -0.79703800 & 1.73396200 \\
\hline $\mathrm{O}$ & 2.04142800 & 1.67829700 & -0.43160500 \\
\hline $\mathrm{O}$ & 3.35072500 & 1.81254100 & -0.03884900 \\
\hline $\mathrm{H}$ & 3.28851500 & 2.20313800 & 0.82442200 \\
\hline \multicolumn{4}{|l|}{$t \mathrm{C}_{8} \mathrm{H}_{17}$} \\
\hline \multicolumn{4}{|c|}{ Cartesian Coordinates $(\AA)$} \\
\hline $\mathrm{C}$ & -4.16426300 & -0.21779900 & 0.08009200 \\
\hline $\mathrm{H}$ & -4.25470100 & -0.40009900 & 1.14765200 \\
\hline $\mathrm{H}$ & -4.25740400 & -1.17489500 & -0.42620800 \\
\hline $\mathrm{H}$ & -5.00583100 & 0.39871800 & -0.22201700 \\
\hline $\mathrm{C}$ & -2.83401300 & 0.45662700 & -0.25420400 \\
\hline
\end{tabular}




\begin{tabular}{lrrr}
\hline $\mathrm{H}$ & -2.78971400 & 0.65577600 & -1.32323100 \\
$\mathrm{H}$ & -2.78788700 & 1.42591100 & 0.23858900 \\
$\mathrm{C}$ & -1.62002000 & -0.37862100 & 0.15599500 \\
$\mathrm{H}$ & -1.67198000 & -1.35001300 & -0.33383600 \\
$\mathrm{H}$ & -1.66590100 & -0.57523800 & 1.22648500 \\
$\mathrm{C}$ & -0.28475200 & 0.28808000 & -0.18010600 \\
$\mathrm{H}$ & -0.24800900 & 1.26337000 & 0.29840500 \\
$\mathrm{H}$ & -0.22989200 & 0.46802100 & -1.25226400 \\
$\mathrm{C}$ & 0.92061200 & -0.55399000 & 0.25282200 \\
$\mathrm{H}$ & 0.80872200 & -1.55736300 & -0.15241400 \\
$\mathrm{H}$ & 0.89200400 & -0.67214800 & 1.34175100 \\
$\mathrm{C}$ & 2.27387200 & -0.01942900 & -0.15179200 \\
$\mathrm{C}$ & 3.41322000 & -1.00219800 & -0.17685400 \\
$\mathrm{H}$ & 3.12350400 & -1.93863300 & -0.64413700 \\
$\mathrm{H}$ & 4.26824300 & -0.60753400 & -0.71778600 \\
$\mathrm{H}$ & 3.75599600 & -1.24054200 & 0.83401300 \\
$\mathrm{C}$ & 2.61635100 & 1.41485200 & 0.15173800 \\
$\mathrm{H}$ & 1.85871800 & 2.10444900 & -0.20427500 \\
$\mathrm{H}$ & 2.71837500 & 1.57809300 & 1.22839300 \\
$\mathrm{H}$ & 3.55972000 & 1.69700700 & -0.30526300 \\
\hline
\end{tabular}

